\title{
Mechanical Properties and Deterioration Instability of Filling Body with Gangue-Cement Material Based on AE Experiment
}

\author{
Guofeng Yu, ${ }^{1,2}$ Yunchun Han ${ }^{\mathbb{D}},{ }^{1}$ Xiaoyang Yu ${ }^{\mathbb{D}},{ }^{3}$ Ren Bao, ${ }^{1}$ Jiaxing Guo, ${ }^{4}$ \\ and Qingbo Feng 4 \\ ${ }^{1}$ State Key Laboratory of Deep Coal Mining \& Environment Protection, \\ Coal Mining National Engineering Technology Research Institute, Huainan 232001, China \\ ${ }^{2}$ School of Energy and Safety, Anhui University of Science and Technology, Huainan 232001, China \\ ${ }^{3}$ A-level International Curriculum Center, Hefei University of Technology, Hefei, 230009, China \\ ${ }^{4}$ North Yansheng Engineering Technology Co., Ltd, Qinhuangdao 066000, China \\ Correspondence should be addressed to Yunchun Han; 353683026@qq.com and Xiaoyang Yu; yuxy23000@126.com
}

Received 16 October 2021; Accepted 8 December 2021; Published 5 January 2022

Academic Editor: Xiao Wang

Copyright (c) 2022 Guofeng Yu et al. This is an open access article distributed under the Creative Commons Attribution License, which permits unrestricted use, distribution, and reproduction in any medium, provided the original work is properly cited.

\begin{abstract}
Gangue materials have been used to solve mine disasters with a support tunnel along the goaf and filling mining. Mastering the properties and damage characteristics of filling materials is an important basis for effective implementation. Based on the conventional uniaxial compression acoustic emission (AE) test, the effects of cementitious materials, ratio between water and cementitious material, gangue particle size, and grading parameters on the mechanical properties of gangue-cement samples were analyzed. The stage characteristics of compression deformation were studied. The fracture propagation characteristics and rock mass failure types induced by different graded gangues were revealed. The fracture forming mechanism from clustered damage and failure was interpreted. The results show that the compressive strength of the backfill increases with the increase of cementitious material; however, it decreases with the increase of water binder ratio. Controlling the proportion and dosage of materials was the key factor to realizing pumpability and stability. Combined with the deformation and AE characteristics, the failure stage of the backfill body is divided into three stages: linear deformation-low energy changing, block compression-high energy changing, and gentle stability-stable energy changing. Affected by the gangue distribution, the load in each stage will induce fracture to produce five distribution modes of single, turning, breakthrough, bifurcated, and collapsed surrounding gangue. In the process of loading failure, different gradation and particle sizes will also change its stress concentration characteristics, resulting in the transformation of rock failure types. The surface structure and roughness of gangue play an important role in the compressive performance of cement paste. The research results try to provide some guidance for efficient filling mining.
\end{abstract}

\section{Introduction}

With the increasing demand of economic development for energy and infrastructure projects, the instability of deep engineering, such as mines and tunnels, has become an important factor restricting safe development. Grouting, filling, and other technologies are widely used in the reinforcement and stability of rock and soil mass [1-5]. Backfilling mining is a green mining technology that supports the roof of goaf and controls surface subsidence by a filling body [6-8]. Filling mining is an important part of green mining that can not only solve the problem of "three under" coal pressure in mines but also effectively control surface subsidence [9-11]. In view of the filling cost and production conditions, filling materials are required to have the characteristics of wide sources, low cost, and effective filling effect $[12,13]$. In structural filling mining of coal mines, the gangue-cemented filling material is used to prepare the filling body according to local conditions. Using the coal mine waste gangue as a filling material can not only control the movement and deformation of the overburden but also reduce the emission of coal mine gangue and reduce the environmental pollution caused by surface wastes, such as gangue. It is 
a filling method with more characteristics of environmental protection, energy conservation, and emission reduction $[14,15]$.

As the main supporting component to prevent mine subsidence, the filling body determines the goaf stability. The strength and size of the filling body are important factors affecting its stability [16-18]. Gangue is a kind of bulk medium belonging to a coarse-grained soil in its particle size composition. A lot of research has been done on the compaction characteristics of granular backfill. According to the theory of soil mechanism, the shape, strength, size, density, gradation, and stress of particles are important factors affecting soil fragmentation [19]. At the same time, to make filling suitable for various mines, multiple types of cemented materials have been used in many mines at home and abroad. In-depth research was conducted on the influencing factors and characteristics of various materials $[20,21]$. According to the research results at this stage, it is considered that the main factors affecting the strength of the filling body are the amount of cement, aggregate particle size composition, water consumption, curing age, mixing time, and curing environment, and the first four factors are the most important.

The filling body is a composite material, and its strength is the interaction between the cement strength, aggregate strength, and other components [22, 23]. The stress-strain curve of the backfill is highly nonlinear before and after the peak stress. On the one hand, this nonlinearity is because of the composite action of materials. On the other hand, it is because of the bonding nature of the cement aggregate. The mechanical deformation characteristics of the filling body are important mechanical properties of the filling body. Only with sufficient understanding of the variation law of the deformation characteristics of the filling body can some mathematical expressions be used to characterize the deformation characteristics of the filling body [24]. The strength of the filling body composed of cement, fly ash, and slag can meet the deformation requirements. The test results show that when tailings and gangue are mixed and filled, adding fly ash to cement can significantly improve the strength of the filling body [25]. How to control the interaction and ratio of various materials has become the key condition to solve the performance of backfill.

As a kind of bulk material, the compression deformation properties are closely related to the particle size and gradation of gangue. The selection of particle size and gradation of gangue materials directly affect the filling effect. Predecessors have carried out a series of studies on filling materials with different particle sizes and gradations. Huang et al. [26] tested the timedependent characteristics of gangue and fly ash filling and established the rheological equation. Xu et al. [27] analyzed the deformation characteristics of gangue fly ash filling material during compaction and studied basic deformation. When the gangue with a smaller particle size accounts for a large proportion in the grading, the compression rate of gangue is small. Liu et al. studied the influence of gangue filling on the roof subsidence of gob retaining roadway and considered that the stiffness of goaf and protection coal and rock mass directly affect the roof subsidence [28]. The deformation and failure of the filling body follow four stages: elastic deformation stage, yield stage, plastic deformation stage, and failure stage. In addition, it is also found from the failure trace of the test block that the distribution of failure fractures depends on the location of the uneven weak points of the test block. Therefore, the fractures show a very irregular state [29]. Li et al. used PFC3D software to simulate the compression deformation of four different particle size grades of coal gangue filling materials [30]. The compression deformation law, particle cluster distribution, and shape change of gangue filling material are analyzed. If the particle size grading ratio is reasonable, large particles form a frame structure, and small particles with different particle sizes can fill the pores of the filling material. Based on the compression test of broken coal gangue under different loading rates, $\mathrm{Li}$ et al. analyzed the antideformation ability, which is the key to controlling overburden movement and surface settlement [31]. Particle breakage will affect the deformation resistance of the filling material. With the increase of loading rate, the damage degree of the sample decreases. The proportion of large-size coal gangue increases, and the $\mathrm{AE}$ activity level gradually increases.

At the same time, the physical and mechanical properties of coal gangue and fly ash are very important for the design of the filling and transportation system. The mineral composition of coal gangue and fly ash affects the strength of the filling body, and the fluidity of slurry affects the mining engineering process [32]. Therefore, according to the requirements of strength and pumpability, Zhang et al. developed a comprehensive green mining technology for coal mining, coal gangue washing and backfilling, rock stratum control, and system monitoring [33]. Huang et al. took the 15061 longwall face of the Dongping coal mine as the research object. The filled coal gangue with different particle sizes and their effects on porosity, stress-strain, strain energy density, and strain were obtained under laboratory conditions. The small particle size of the backfill (coal gangue) will reduce porosity and improve strength. However, the cost of backfill will increase, which must also be taken into account when determining the material specification [34]. These studies are only aimed at the elaboration of experimental phenomena and the determination of reasonable gangue particle size and gradation by experiments without further analysis and discussion. Therefore, Chen et al. studied the influence of red mud, chloride, and other materials on the strength of coal gangue-cemented paste filling from a micro point of view based on mechanical experiments and electron microscope scanning $[35,36]$. Li et al. used design expert and central composite design (CCD) software to analyze the effects of fly ash content, fine gangue ratio, mass concentration, and the effects of mass concentration, fly ash content, and fine gangue ratio [12]. Therefore, it is very important to accurately grasp the influence of coal gangue and fly ash on the strength of backfill.

As a nondestructive testing technology, AE is increasingly used to study the mechanical properties of rock mass materials [37-39]. Under the condition of normal temperature curing, the $\mathrm{AE}$ characteristics of the rock mass in the process of load failure show a certain law [40]. Stress relaxation will occur when fractures are formed because of the failure of the rock mass structure. As the energy 
accumulated by deformation will be suddenly released in the form of an elastic wave, this phenomenon is called AE. A large number of test results show that by analyzing the $\mathrm{AE}$ signal of the stress process of rock mass, the evolution of microfractures in the structure and the study of failure mechanism can be effectively inferred [41, 42]. Li et al. introduced the $\mathrm{AE}$ system into the conventional confined compression test. During the loading process, the filling will have particle breakage, which has a significant impact on its deformation resistance. The gangue block will be accompanied by obvious $\mathrm{AE}$ characteristics in the process of sliding, overturning, and failure. Under the condition of constrained compression test, monitoring AE parameters can reveal the influence mechanism of particle breakage on deformation [43]. Qi et al. analyzed the changes of these characteristics with time using stress-strain resistivity and $\mathrm{AE}$ tests and studied their microscopic properties. AE can reflect fracture initiation and propagation and explain the change of sample resistivity under uniaxial loading [44]. Therefore, the failure mechanism of the filling body can be analyzed based on AE technology, which provides the important basic support for efficient filling mining.

To reveal the mechanical properties and failure characteristics of the gangue-cement backfill, the influence of cementitious materials and gangue grading parameters were analyzed using the conventional uniaxial compression $\mathrm{AE}$ test. The failure characteristics and fracture propagation law under stress would be explained. Then, it will provide some theoretical support for mine safety mining.

\section{Experimental Methodology}

\subsection{Filling Materials}

2.1.1. Cement. The main types of cement suitable for cemented filling in mines are ordinary Portland cement, slag Portland cement, and pozzolanic Portland cement [45]. The density of ordinary Portland cement is $3.0 \sim 3.15 \mathrm{t} / \mathrm{m}^{3}$, usually $3.1 \mathrm{t} / \mathrm{m}^{3}$, and the unit weight is $1.0 \sim 1.6 \mathrm{t} / \mathrm{m}^{3}$, usually $1.3 \mathrm{t} / \mathrm{m}^{3}$. The density of slag cement and pozzolanic cement is $2.85 \sim 3.0 \mathrm{t} / \mathrm{m}^{3}$, usually $2.9 \mathrm{t} / \mathrm{m}^{3}$, and the unit weight is $0.8 \sim 1.15 \mathrm{t} / \mathrm{m}^{3}$, usually $1.1 \mathrm{t} / \mathrm{m}^{3}$. The hydration of cement starts from the surface of particles. The larger the specific surface area of cement, the faster the hydration and the faster the formation strength.

2.1.2. Fly Ash. Fly ash is a fine powder collected from the boiler flue gas of pulverized coal-fired thermal power plant, also known as fly ash. Its composition is similar to that of high alumina clay and mainly exists in a vitreous state [46]. Fly ash is a kind of pozzolanic mixed material formed by the hightemperature combustion of pulverized coal. It has little or no hydraulic cementitious properties. However, when it coexists with water in powder form, it reacts with calcium hydroxide or other alkaline metal hydroxides to form compounds with hydraulic cementitious properties and becomes a material to increase strength and durability. Fly ash is added to the filling material to improve the strength of the filling body, and fly ash is used to replace part of the cement. In a high concentration or paste filling slurry, the existence of an appropriate amount of fly ash can reduce the pipeline transportation resistance and improve the pumping performance of paste. Among the physical properties of fly ash, fineness and particle size are more important items, which directly affect other properties of fly ash. The finer the fly ash, the greater the proportion of fine fly ash and its activity. The fineness of fly ash affects the early hydration reaction, while the chemical composition affects the later reaction.

2.1.3. Gangue. The physical characteristic parameters of gangue have a great influence on the technology of cemented filling and the quality of the filling body. The particle size characteristics of the gangue have a great influence on the strength index, working characteristics, and process of cemented filler. In general, the particle size of the gangue has a more significant impact on the cemented filler than the aforementioned factors, such as mineral component content, physical properties, and mechanical indexes of gangue. The particle size composition of the gangue refers to the natural grading or artificial crushing grading of the waste rock constituting filling materials. This grading characteristic not only depends on the structural characteristics, such as rock mass, rock joints, and fissures, and mechanical indexes, such as strength, but also has a very important relationship with the production technology of gangue. For naturally graded excavation gangue, it will depend on the structural characteristics of the rock mass, blasting technology, and blasting parameters. The artificial grading of the crushed gangue mainly depends on the mechanical properties of the rock and the crushing process:

(1) Particle size: the gangue is screened with screens containing different apertures. The pore size of the screen is $5,10,15,20$, and $25 \mathrm{~mm}$, respectively. Six groups of single particle size gangues with particle sizes of more than $0-5,5-10,10-15,15-20,20-25$, and $25-30 \mathrm{~mm}$ can be obtained by screening.

(2) Discontinuous gradation: in particle research, $5 \mathrm{~mm}$ is generally used as the boundary to divide into coarse and fine particles. It is generally believed that coarse materials form a skeleton. The finer the material, the better the filling. The smaller the gap between the filling particles, the smaller the compression deformation of the filling body. To study the influence of different fine content on the compression characteristics of the gangue, the percentage of fine content in the sample is set as $10 \%, 20 \%, 30 \%$, $40 \%$, and $50 \%$, respectively.

(3) Continuous gradation: at present, the Fuller theory and Talbot theory are widely used in continuous grading theory. The Fuller theory holds that the closer the particle grading curve to the parabola, the greater the density and the smaller the porosity. Taylor's theory holds that Fuller's formula is an ideal grading curve, and there is a certain fluctuation range to obtain the maximum density in practice. According to the Taibo formula [47], the 
experimental analysis is carried out with the coefficients of $0.3,0.4,0.5,0.6$, and 0.7 , respectively.

(4) Water: reasonable water consumption can improve the mechanical strength of the backfill without increasing the cost. Therefore, the use of optimal water consumption is often an important factor in cost saving. For the cemented filler with certain requirements for transportation performance, the role of water is not only to hydrate the cement but also to make the filler meet certain transportation performance. Therefore, water consumption is often greater than the water required to obtain the maximum mechanical strength value. The waste rockcemented filling generally adopts a separate transportation process of aggregate and slurry, which has no special requirements for transportation performance. The only goal of water consumption is to obtain the maximum cementation strength. Therefore, for the waste rock-cemented filling process, the most important thing is to determine the best water consumption that can obtain the maximum mechanical strength of the filling body.

2.2. Filling Sample. The different particle size, gradation, and particle shape of filling materials have a great impact on the strength index, compactness, and flow state of the two-phase flow. Gangue aggregate is an important factor affecting the strength of the backfill, except cement. The well-graded gangue aggregate shall be the aggregate with the minimum porosity and the maximum compactness, and it can ensure good bearing characteristics and necessary permeability to minimize the loss of the fine particle composition of the filler. The coarse aggregate may or may not be added to the filling material of the mining filling body. Under the condition that the material gradation meets the conditions and the mix proportion is determined, increasing the proportion of coarse aggregate will improve the permeability of the slurry and be conducive to the formation of the early strength of the backfill. Excessive fine aggregate content consumes more cementitious materials. It is not only conducive to the penetration of excess water in the filling slurry but also reduces the strength of the filling body. According to the test requirements and purposes, the main pouring materials used are shown in Table 1.

The cylindrical uniaxial compression AE test was carried out. After the test piece is placed around the injection mold for 24 hours, the mold shall be removed and placed in the curing room for curing (normal temperature, humidity 95\%). After the gangue-cement sample reaches the predetermined age $(1 \mathrm{~d}, 4 \mathrm{~d}, 7 \mathrm{~d}$, and $14 \mathrm{~d})$, the AE sensors are arranged on the surface of the cylindrical specimen. Relevant experimental research is carried out by the testing machine, and the gangue-cement samples are shown in Figure 1.

\subsection{Experimental Design}

2.3.1. Experimental Method. The uniaxial compressive strength test of the rock is the load per unit area when the specimen is damaged by the axial pressure under unconfined conditions. After the curing cycle of coal gangue specimens is completed, various coal gangue specimens shall be obtained, firstly, for the $50 \mathrm{~mm} \times 50 \mathrm{~mm} \times 100 \mathrm{~mm}$ cylindrical test piece. Then, carry out experimental research on the testing machine. In addition to the strength data of the filling cement, the strain data should also be obtained. Therefore, the strain gauge is used to test the strain data of the specimen during loading to observe the deformation characteristics of the cement. To eliminate the end effect under load, steel cushion blocks are placed at both ends of the specimen. The diameter of the cushion block is equal to or slightly larger than the diameter of the test piece, and the stiffness and flatness of the cushion block shall meet the requirements of the pressure bearing plate. To ensure the smooth progress of the test, the test shall be carried out in strict accordance with the test operation procedures to ensure the accuracy of the test data and make the test successful [48]. The loading process is as follows:

(1) Prepare the cured specimen, grind the end flat, and stick a strain gauge to measure its strain value.

(2) Select the measuring range according to the test piece, hang the thallium and align the scribed line

(3) Adjust the buffer valve to adapt to the measuring range. (4) Turn the main switch to turn on the power supply.

(5) Start the motor of the oil cylinder, unscrew the oil feeding valve, raise the piston up for a period, and adjust the pointer to stop the oil pump motor after zero.

(6) Start the loading speed indicator motor and quickly adjust it to the appropriate position. At this time, the indicator panel rotates at a certain speed.

(7) Place the test piece at the center of the press-bearing plate and adjust the base to make the stress on the test piece uniform up and down.

(8) Start the oil pump motor, quickly adjust the handle of the oil supply valve to the corresponding position, and load it at the speed of $0.05 \mathrm{~mm} / \mathrm{s}$ until the test piece is crushed.

(9) Record the test value.

(10) Open the oil return valve and pull back the driven needle.

(11) Turn off the loading speed indicator knob.

(12) Remove the crushed test piece. The test process is as follows: firstly, prepare the cured specimen and install the strain gauge and $\mathrm{AE}$ sensor to measure its strain value and AE signal. Then, start the loading system and signal the acquisition system. Place the test piece at the center of the press-bearing plate. Load at the speed of $0.05 \mathrm{~mm} / \mathrm{s}$ until the test piece is crushed. During the test, the test value and $\mathrm{AE}$ signal characteristics shall be recorded in real time.

Six AE sensors are staggered on the surface of the sample. The sample to be tested between the emission source and the 
TABLE 1: Main artificial materials of making the sample.

\begin{tabular}{|c|c|c|c|c|c|c|c|c|}
\hline Gangue material & & & & & & Cement & Fly ash & Additive \\
\hline Particle size $/ \mathrm{mm}$ & $0-5$ & $5-10$ & $10-15$ & $15-20$ & $20-25$ & \multirow{2}{*}{$\begin{array}{l}\text { Portland } \\
\text { cement of } \\
32.5\end{array}$} & \multirow{2}{*}{$\begin{array}{l}\text { Class II fly ash: the residue of } 0.045 \mathrm{~mm} \\
\text { square hole sieve is less than } 20 \% \text {, and the } \\
\text { burning vector is less than } 8 \%\end{array}$} & \multirow{2}{*}{$\begin{array}{l}\text { Early strength } \\
\text { agent plaster }\end{array}$} \\
\hline Continuous coefficient & 0.3 & 0.4 & 0.5 & 0.6 & 0.7 & & & \\
\hline $\begin{array}{l}\text { Discontinuous } \\
\text { coefficient }\end{array}$ & 10 & 20 & 30 & 40 & 50 & & & \\
\hline $\begin{array}{l}\text { Ratio between water and } \\
\text { cementitious material }\end{array}$ & & 1.7 & & .8 & & 1.9 & 2.0 & 2.1 \\
\hline $\begin{array}{l}\text { Cementitious material/ } \\
\mathrm{kg} \cdot \mathrm{m}^{-3}\end{array}$ & & 50 & & 00 & & 150 & 200 & 250 \\
\hline
\end{tabular}

Conventional proportion: the proportion of water, cement, fly ash, and gangue waste is $1.3: 1: 1: 9$. Cast a cylindrical sample of $50 \mathrm{~mm}{ }^{*}$ $100 \mathrm{~mm}$

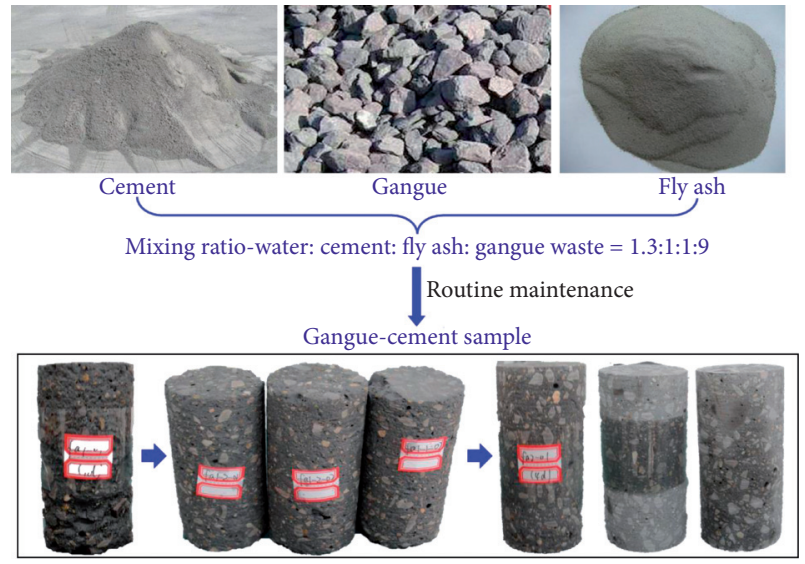

Figure 1: Gangue-cement sample.

receiver was placed, and use Vaseline to couple the probe with the sample. The damage and fracture of the rock mass during the application of external load on the sample can be monitored, and the evolution form of macro fractures was analyzed as shown in Figure 2. After the rock mass is subjected to an external load, the strain energy stored in the rock mass will be released in the form of an elastic wave to produce sound. AE signals can be detected, recorded, and analyzed by instruments. The signal wave can be used to infer the AE source. Combined with the acoustic source information, the rock failure characteristics can be obtained.

2.3.2. Wave Velocity Test Method. To carry out AE research, the equipment used in this experiment is the TH-F ultrasonic testing system to measure its wave velocity. The system mainly consists of a transmitting source, transducer, receiver, oscilloscope, and microcomputer (as shown in Figure 2). The specific operation method of this experiment is as follows: place the sample to be tested between the transmitting source and the receiver, and use Vaseline to couple the probe with the sample. Then, turn on the emission source and send an ultrasonic wave (incident wave) to the sample to be tested. After the ultrasonic wave is received by the receiver through the sample to be tested, it is received, displayed, and stored (transmitted wave) by the oscilloscope. The transmitting source and the receiver are in direct contact, and the contact surface is coupled with Vaseline. Then, turn on the transmitting source and send ultrasonic waves to the receiver. The time required for the ultrasonic wave transmitted by the transmitting source to propagate to the receiver is the delay time of the system itself. Determine the onset time of the incident wave, transmission wave, and the delay time of the system itself. The calculation formula of ultrasonic wave velocity in the test sample can be obtained.

$$
c=\frac{L}{T-T 0}
$$

where $c$ represents the ultrasonic wave velocity measured by the sample; $L$ is the length of the sample to be tested; $T$ is to determine the initiation time of incident wave; and $T_{0}$ is the delay time of the electronic circuit itself, which is determined as $7 \mu$ s by an indoor test.

\section{Results}

The main factors affecting the compressive strength of the gangue are cement dosage, water to cement ratio, aggregate gradation, strength, interfacial bonding ability between cement and aggregate, and curing environment. The main reasons for the low compressive strength of the filling body are as follows: (1) the content of cement is low, and the products of hydration reaction and pozzolanic reaction cannot effectively fill the pores in the samples. (2) The gangue with medium and coarse particle size acts as an aggregate in the filling material, and they form a support network system with each other.

\subsection{Mechanical Properties}

3.1.1. Compressive Strength of Rock Mass under Slurry Control. The stress-strain curves of the gangue-cement samples in different curing cycles are obtained by the AE-SS experiment. The typical test results are shown in Figure 3, and the strength of the cement at $1 \mathrm{~d}, 4 \mathrm{~d}, 7 \mathrm{~d}$, and $14 \mathrm{~d}$ is obtained. It can be found that the compressive strength of cement increases significantly with the increase of the curing cycle. The early strength $(4 \mathrm{~d})$ can reach $0.76 \mathrm{MPa}$, while the later strength $(14 \mathrm{~d})$ can reach $7.4 \mathrm{MPa}$. Of course, because of the influence of material parameters, such as gangue and fly ash, there are certain differences in the strength variation 


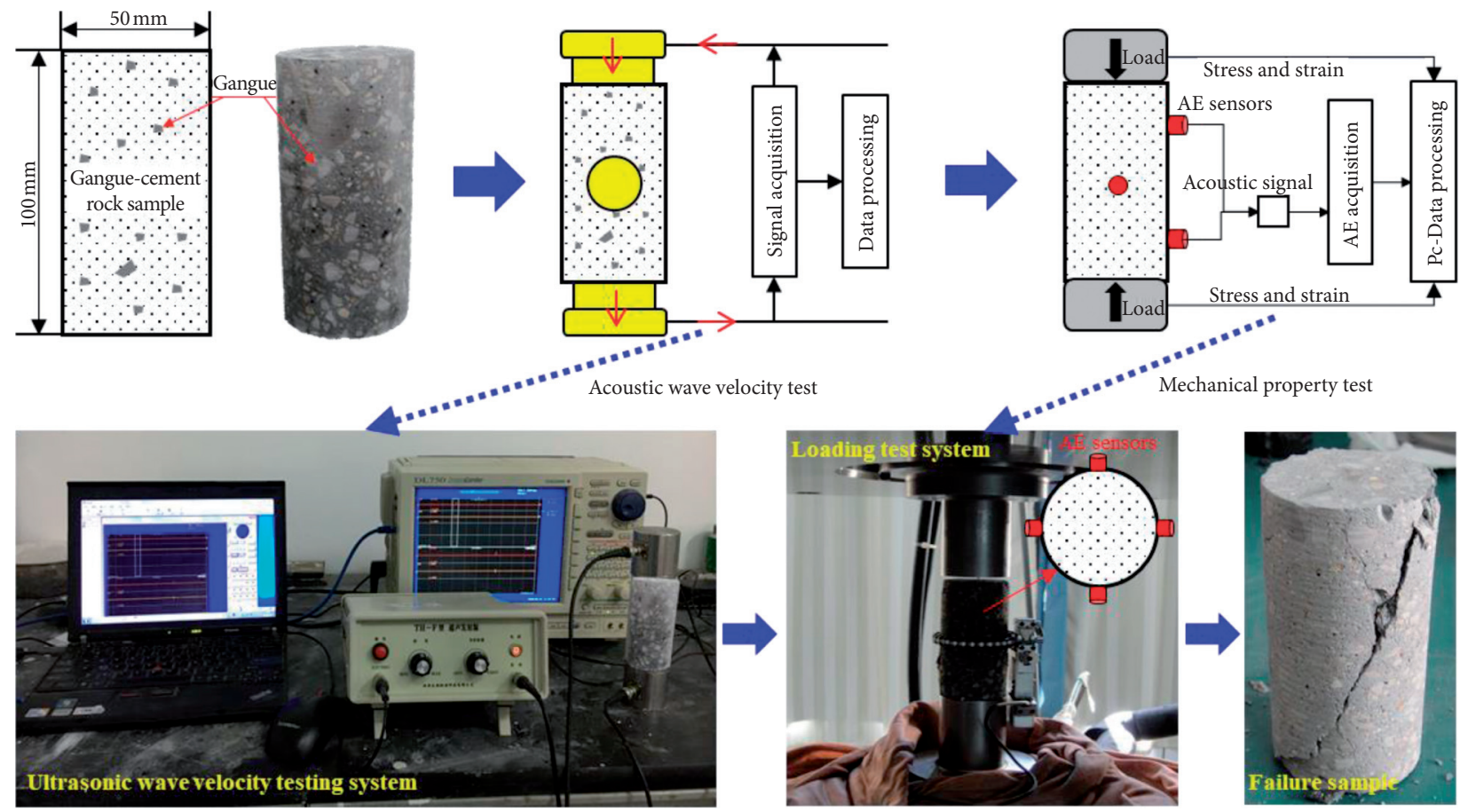

FIgURE 2: AE and stress-strain (AE-SS) experiment.

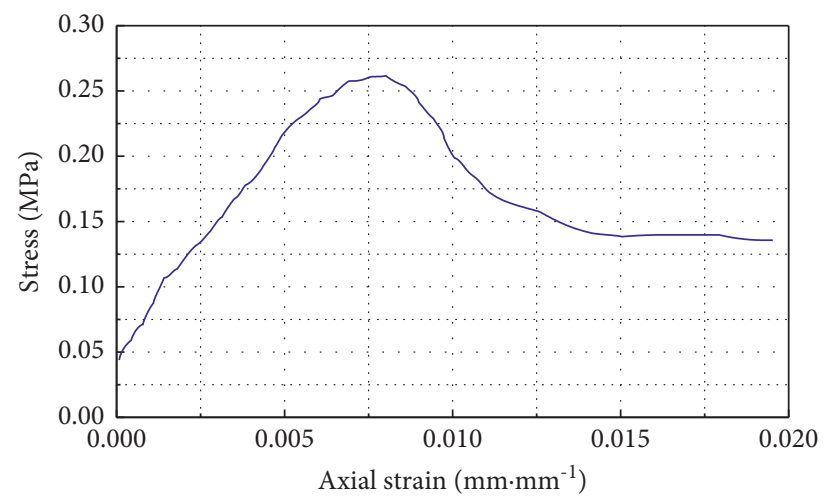

(a)

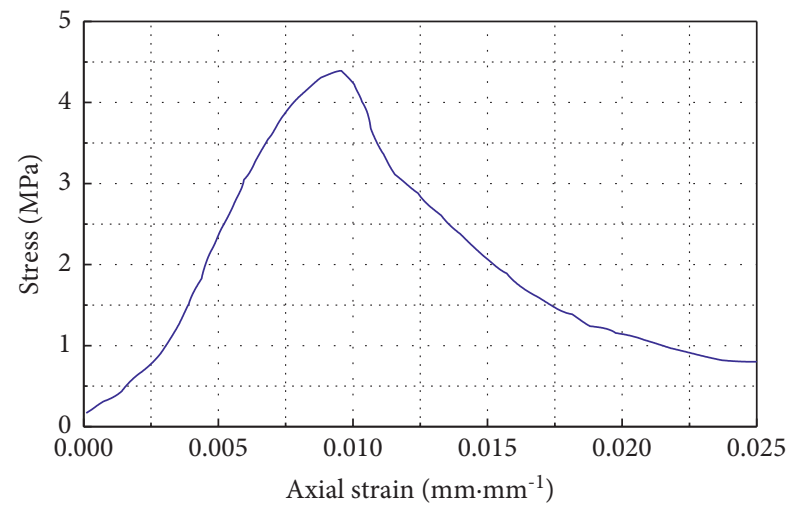

(c)

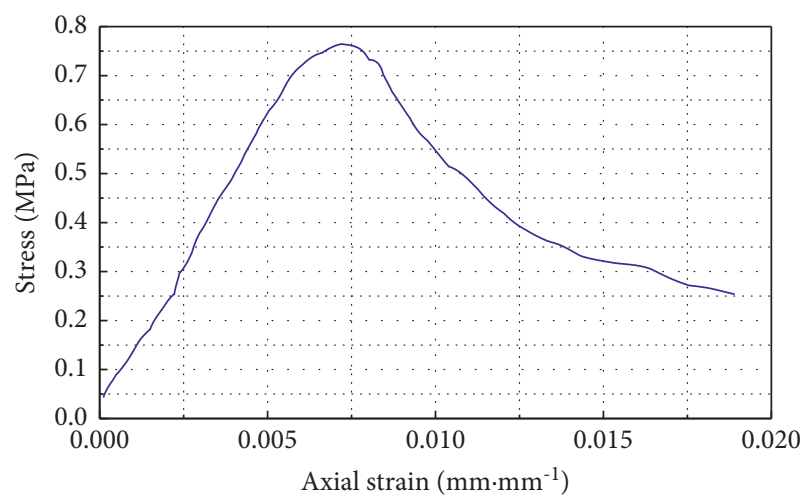

(b)

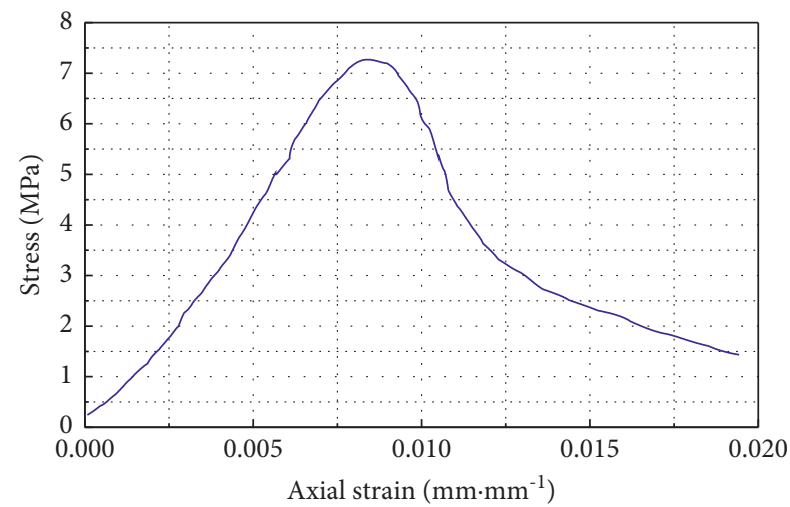

(d)

FIgURE 3: Compressive strength of gangue-cement sample. (a) Stress-strain curve of the 1-day sample, (b) stress-strain curve of the 4-day sample, (c) stress-strain curve of the 7-day sample, and (d) stress-strain curve of the 14-day sample. 
characteristics of the filling body, and the final strength will also be different. To analyze the influence of various factors on the early strength of the filling cement, multiple groups of uniaxial compression tests are continued and analyzed by the multiple linear regression method.

(1) Influence of Cementitious Material Dosage. When the ratio between water and cementitious material (R-WCM) is fixed at 1.8, the amount was adjusted to analyze the relationship between the early strength and the cementitious material. The results are shown in Figure 4. It can be seen from Figure 4 that the strength of the filling body increases linearly with the increase of cementitious material. Under the same dosage of cementitious material, its early strength will show a large growth rate, and the growth rate will slow down in the later stage. Moreover, with the increase of the amount of cementitious material, the final strength will be significantly improved. However, the increase of cementitious material consumption will increase the cost of filling and mining. The dosage of cementitious materials shall be determined according to the strength requirements in combination with the actual situation.

The reason is that it is greatly affected by the interaction between cement and fly ash. With the same cement content, the strength increases continuously with the increase of curing age. The effect of intensity growth is the most obvious from 7 to 14 days. This may be because of the large amount of fly ash, and the strong hydration process of the cement is delayed by 7 to 14 days. At the same time, the pozzolanic effect of fly ash plays a role. The hydration of cement and the pozzolanic effect of fly ash play a role at the same time, resulting in a large number of cementitious bodies, which greatly increases the uniaxial compressive strength of the paste backfill. At the same time, the ratio of cement to fly ash and the water binder ratio are also the factors affecting the uniaxial compressive strength of the filling paste. There should be an optimal proportion relationship between cement and fly ash. Under this proportional relationship, the hydration degree in the filling paste reaches the highest, and the compressive strength of the filling paste is the highest. The more the fly ash is added, the more obvious the later strength change is. With the same amount of fly ash, the compressive strength increases gradually with the increase of curing age. The more the fly ash is added, the more obvious the increase of compressive strength is. The amount of fly ash has little effect on the early strength of the paste backfill, which mainly plays the role of micro aggregate. The effect on the middle and later strength is very obvious, and the greater the amount of fly ash, the more obvious the effect.

(2) Effect of R-WCM. R-WCM represents the ratio of the mass of water to the mass of cementitious material. The R-WCM cannot be replaced by the mass concentration because the mass concentration reflects the mass fraction of the solid part in the total mass of the slurry. When the mass concentration is the same, the R-WCM may be inconsistent. Hence, there is a difference between the two. While keeping the mass fraction of the coal gangue, fly ash, and tailings unchanged, adjust the water binder ratio of the paste slurry, study the early strength changes under the action of different water binder ratios, test the strength values corresponding to different water binder ratios, and draw a curve as shown in Figure 5 .

It can be seen from Figure 5 that the early strength of the filling cement decreases with the increase of R-WCM. It is because with the increase of R-WCM, the void ratio in the filling cement body is increasing, resulting in the decrease of the strength of the filling cement body. When the R-WCM is relatively small, the early strength of the filling cement is large. However, the R-WCM cannot be reduced blindly because the reduction of R-WCM will reduce the slump and make it difficult to transport the slurry.

3.1.2. Deformation. The characteristics of gangue grading are varied with different particle sizes. The influence of the filling paste aggregate, i.e., the coal gangue, on the strength performance of the paste filling is mainly because of different particle size gradations. Under good grading conditions, the slurry in the filling paste is evenly distributed, the porosity is small, and the uniaxial compressive strength is high. On the contrary, the slurry inside the filling paste is insufficient, the porosity is large, and the uniaxial compressive strength is low. Therefore, aggregate gradation has a very important influence on the uniaxial compressive strength and strength development of the filling paste. Under the conditions of certain cement dosage, fly ash dosage, paste slurry mass concentration and coal gangue dosage, and without additives, the uniaxial compressive strength of the specimen increases at first and then decreases with the increase of fine gangue rate in the same age, and the early and late laws are the same. The reason is that if the rate of fine gangue is too small, the content of coarse aggregate is too large, and the fine aggregate filled into the gap is insufficient, resulting in large voids and low strength of the formed paste. With the ratio increasing, the fine aggregate filled into the pores increases, the porosity of the paste specimen decreases, and the strength increases. The rate of fine gangue continues to increase, and the overall specific surface area of the paste also increases. When the cement content is certain, the slurry is seriously insufficient. The chemical condensation produced by the hydration reaction of the cement particles and the physical condensation between the cement particles will be weakened. Hence, the strength will also be reduced. The fine gangue ratio has a long-term effect on strength. It is mainly because the integrity of the filling paste is improved under the cementation of cement and fly ash in the later stage so that the overall strength of the filling body is improved.

Furthermore, the compression deformation curve of the gangue is defined as the corresponding relationship between the vertical pressure of the gangue and the amount of compression deformation. Three groups of gangue-cement samples of each particle size and gradation are taken for the test. The corresponding relationship between the vertical pressure and compression deformation of gangue with each particle size and gradation is calculated. Then, the relationship curves of the vertical pressure compression deformation of the gangue with different particle sizes and 

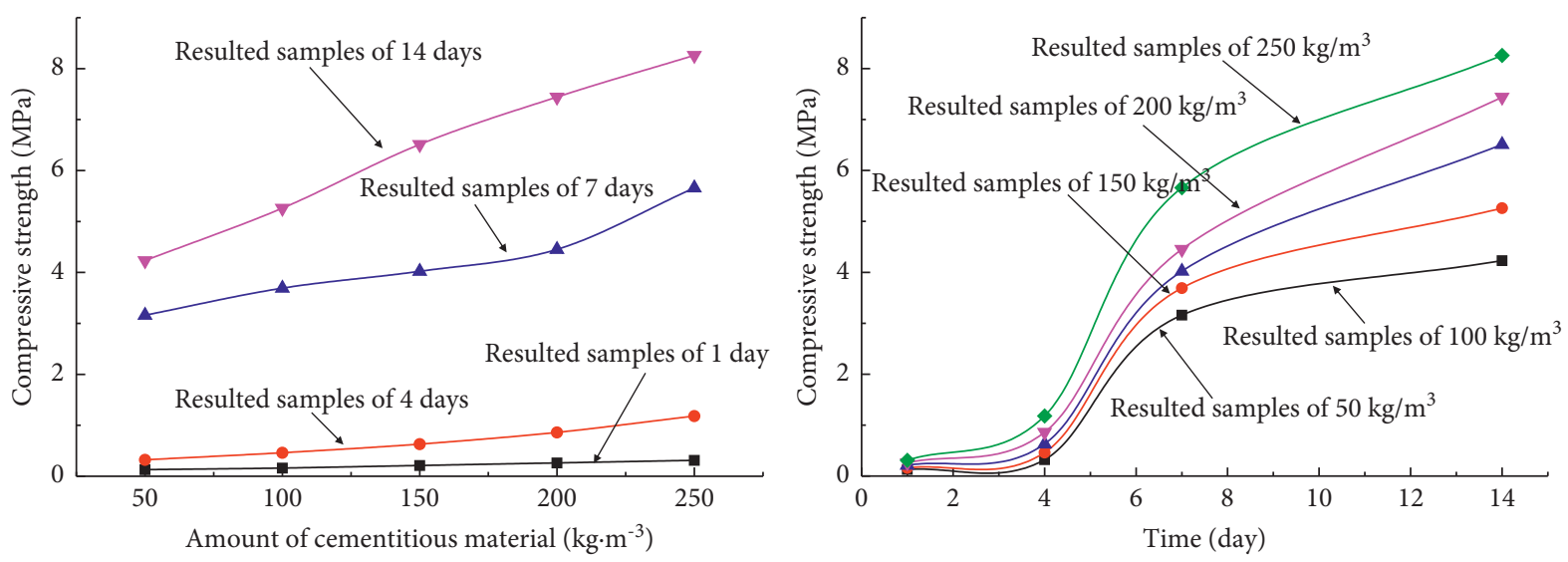

FIGURE 4: Early strength with the amount of cementitious material.
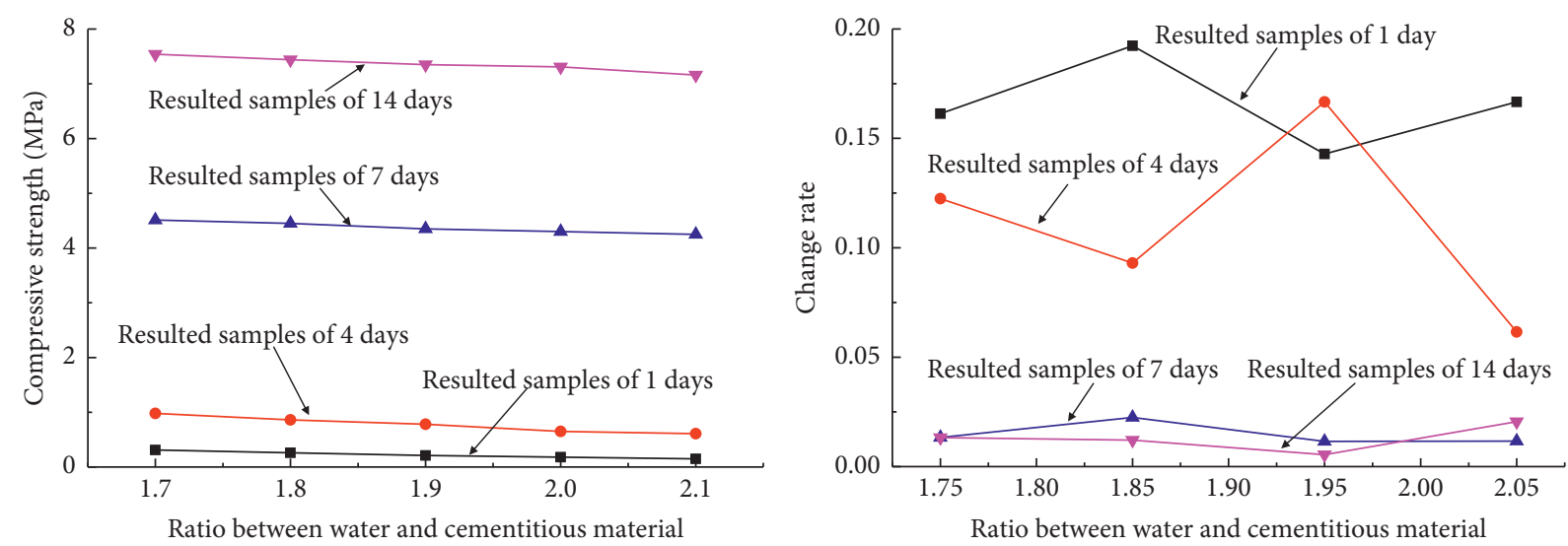

FIGURE 5: Early strength with R-WCM.

grading are drawn. The compression curves of single particle size gangue, discontinuous graded gangue, and continuous graded gangue are shown in Figures. 6-8.

(1) Deformation with the Single Particle Sizes. The compression curve of a single particle size gangue filling material roughly has three stages. The first stage is the linear deformation stage. At the initial stage of the gangue compression, the gangue is continuously compacted, and the gap between the gangue particles is rapidly compressed. The compressive deformation of the gangue increases linearly with the increase of vertical stress. In this stage, the broken gangue dispersion is in a loose state. There are a lot of pores between the gangue particles, which lead to their lesser ability to resist deformation. When subjected to axial external force, the contact state between the particles will be readjusted. The pores of gangue are filled, showing the increase of axial pressure. The axial deformation increases rapidly, and the axial stress and strain are linear. From the perspective of energy consumption, a small part of the energy consumed in this stage is dissipated in the work required for the fracture of the stress concentration part of the gangue. Most of the energy is used to overcome the friction resistance of the gangue moving to the gap.
The second stage is transformed into a logarithmic form. In this stage, after the first stage, the gap between the gangues is greatly reduced, and the density increases. The compression deformation of the gangue is no longer as easy as that in the first stage. To make the gangue produce greater compression deformation, the vertical pressure must be increased. It makes the gangue to be crushed constantly under great pressure. The crushed gangue further fills the small voids, thus increasing the density of the gangue. The compression deformation amplitude of the gangue decreases, and the compression deformation curve of the gangue presents a logarithmic form. With the increase of axial stress, the pore filling effect between gangue dispersions decreases gradually. The damage of the large particle gangue increases obviously. The strength and frequency of the gangue fracture are greatly improved. As most of the pores between the gangue particles are gradually filled, there are no large pores between the particles. A gangue dispersion is gradually transformed into a honeycomb structure with a strong bearing capacity. With the further increase of the axial load, the compaction process of the gangue changes from pore filling to fracture compaction and focuses on fracture compaction. 


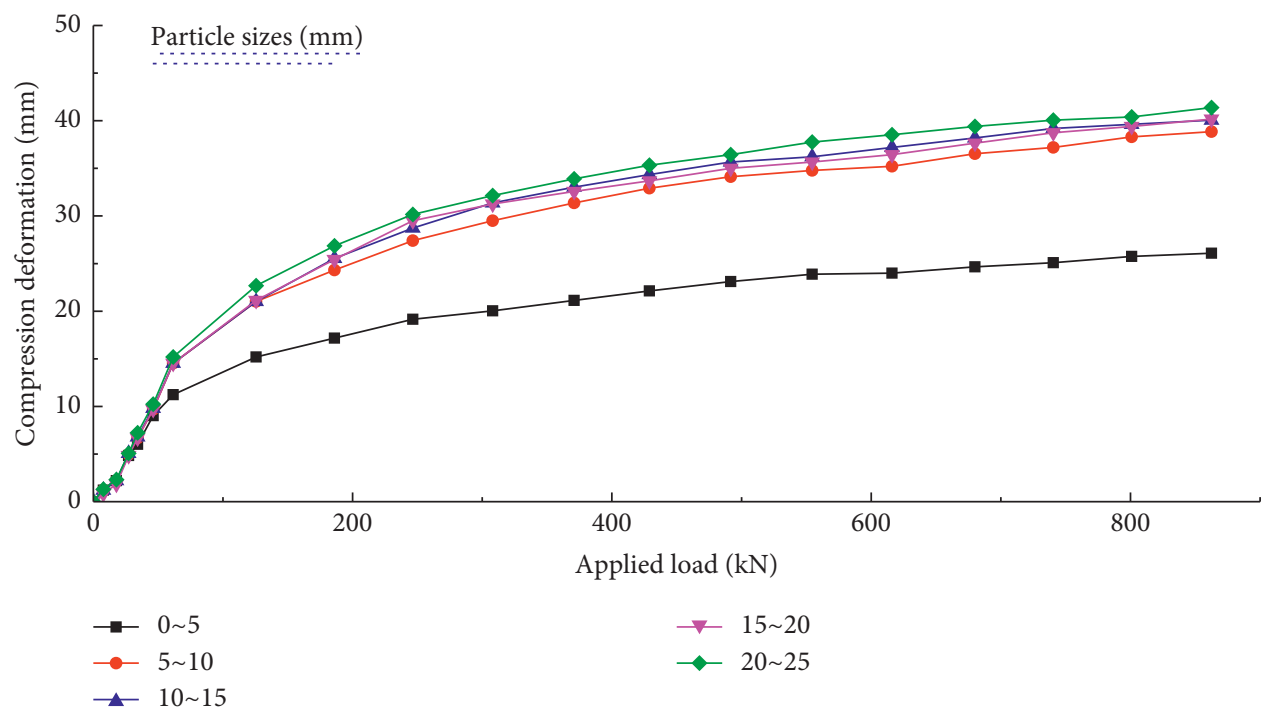

FIGURE 6: Deformation characteristics of gangue-cement samples under different particle sizes.

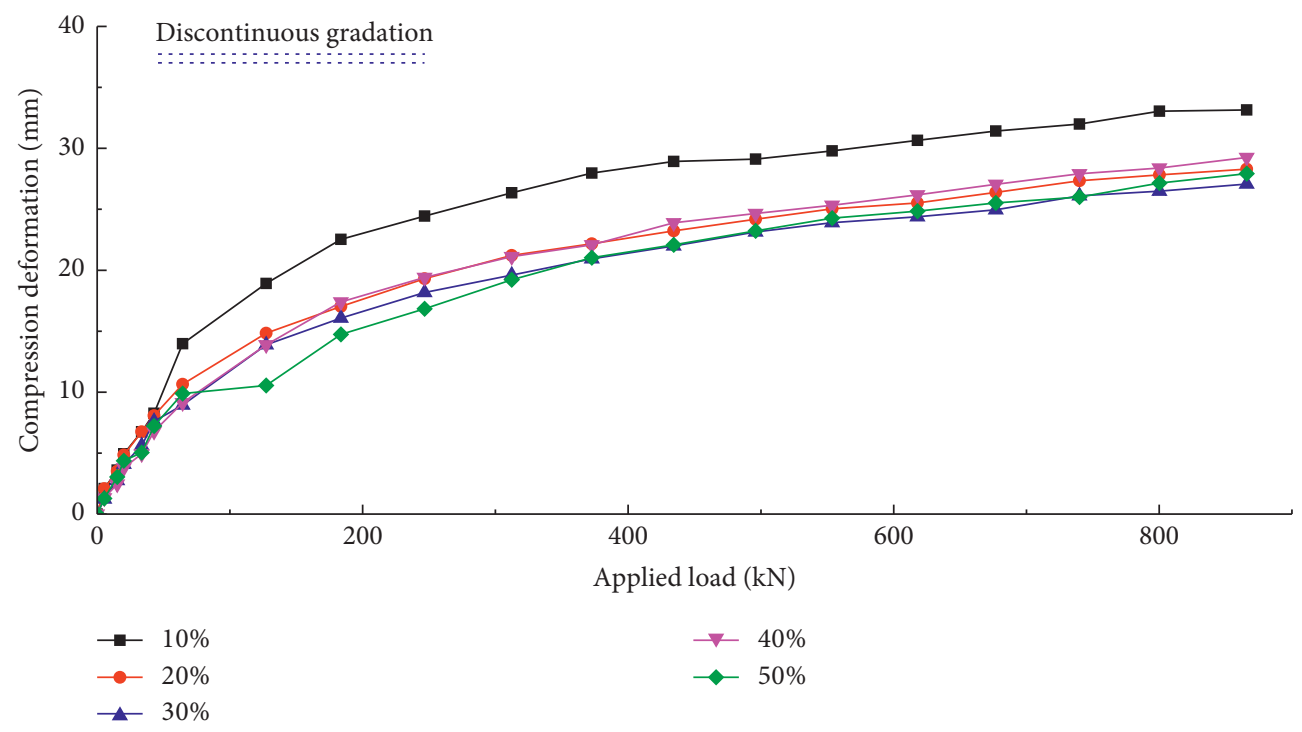

FIGURE 7: Deformation characteristics of gangue-cement samples under discontinuous gradation.

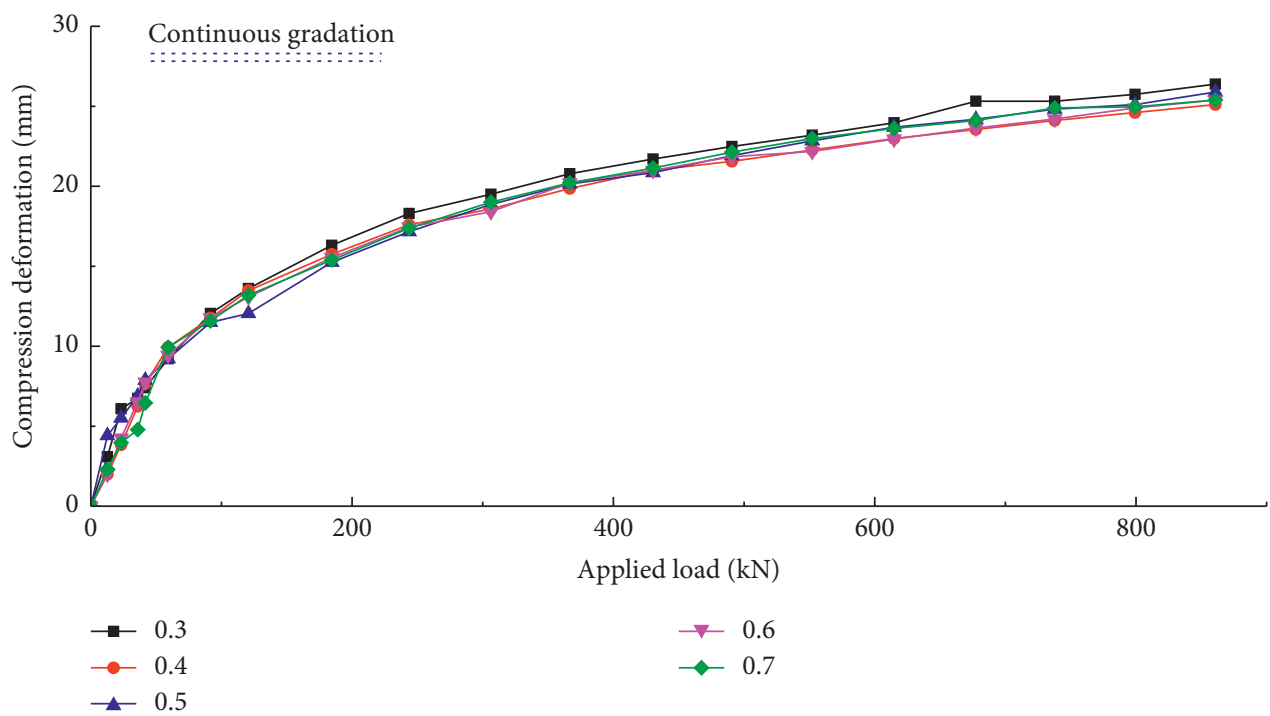

FIGURE 8: Deformation characteristics of gangue-cement samples under continuous gradation. 
The characteristic of the third stage is that the compression deformation of the gangue tends to be stable in a linear manner with the increase of stress. After the second stage compression deformation of gangue, the gap between the gangues is further enriched. The deformation characteristics of the gangue are gradually transformed from the characteristics of the bulk medium to the characteristics of the continuous medium. Therefore, when the vertical pressure increases further, the compression deformation range of the gangue becomes smaller and smaller, and gradually, it presents the characteristics of a continuous medium. The axial stress increases rapidly in this stage. The axial strain growth is small or unchanged. When the compression reaches this stage, the bulk characteristics of the gangue disappear and show the characteristics of a complete elastomer.

Under the same vertical pressure, the compression deformation of the gangue with large particle size is large. The final compression of $0-5 \mathrm{~mm}$ single particle size gangue filling material is significantly less than that of other particle sizes. The smaller the particle size of the gangue filling material, the faster the compression deformation curve tends to be stable. It is mainly because of the relatively high void ratio between the large-size gangue particles. The gangue material has a large compression space in the compression process. In the first and second stages of the compression deformation curve, the compression deformation curves of the gangue filling materials with various particle sizes coincide. It is because the first two compression deformation stages are mainly caused by the compression of the gap between gangue dispersions and the crushing of the gangue.

(2) Deformation of Discontinuous Graded Gangue Filling Material. According to the experimental parameters, the deformation mechanism of the filling body under the condition of discontinuous gradation is obtained as shown in Figure 7 . When the fine material is less than $10 \%$, the discontinuous graded gangue filling material will still have the first stage compression deformation. It shows that when the fine material is less than $10 \%$, the fine material is not enough to fill the gap of the coarse material. Hence, there will still be a gap compression deformation in the first stage. When the content of the fine material exceeds $20 \%$, the gap compression deformation in the first stage gradually disappears because the gap between the coarse materials is filled with fine material.

(3) Deformation of Continuous Graded Gangue Filling Material. According to the experimental parameters, the deformation mechanism of the filling body under the condition of continuous gradation is obtained as shown in Figure 8 . The compression deformation curves of each continuously graded gangue filling material have little difference. The compression performance of a continuously graded gangue filling material is more stable than the first two. As the voids between the gangue particles with different sizes can be filled by gangue particles with different sizes, the first stage the deformation curve of a continuously graded gangue filling material is not obvious, i.e., there is no obvious void compression stage.
The compression of gangue backfill can be roughly divided into three stages: the first stage is the linear deformation stage. At the initial stage of compression, the gangue is continuously compacted, and the compressive deformation of the gangue increases linearly with the increase of vertical stress. The second stage is the block compression stage. This stage is mainly because of the deformation and damage of gangue block, and the crushed gangue further fills and compacts the small voids. The characteristic of the third stage is that the compression deformation of the gangue tends to be stable in a linear manner with the increase of stress. After the second stage compression deformation of the gangue, the gap between the gangues is further enriched. The deformation characteristics of the gangue are gradually transformed from the characteristics of a bulk medium to the characteristics of a continuous medium. Hence, the compression deformation amplitude of the gangue is becoming smaller and smaller.

3.2. AE-SS Analysis. According to the experimental design scheme, the AE monitoring system is installed in the mechanical experiment of each gangue-cement sample. During the loading process, the measured wave velocity is used to obtain the AE signal of the rock fracture. Furthermore, the variation characteristics of stress and $\mathrm{AE}$ with time under the grading conditions of the gangue with different particle sizes in different curing times are obtained as shown in Figure 9.

In the early stage of loading, the pores between the gangues are compacted because of external pressure, and the gangue is broken less. Rock signals mainly come from friction AE. Hence, the energy changes little. In the middle stage of loading, the gangues squeeze each other, resulting in relative sliding. There are many broken gangues. The signals are composed of friction $\mathrm{AE}$ and crushing $\mathrm{AE}$, and the energy changes greatly. In the later stage of loading, the gangue finally forms a relatively stable compacted body under the action of external pressure. The main source of the AE signal becomes the friction type again, and the energy is relatively stable. At the same time, with the increase of the proportion of large particle size gangue, the AE energy increases gradually. The energy of large particle size gradation is greater than that of small particle size gradation. It shows that the energy produced by the crushing of a large particle size gangue is more than that of a small particle size gangue. The $\mathrm{AE}$ parameters in each stage are the highest under the grading condition of the large particle size and high proportion. It shows that the $\mathrm{AE}$ activity is more frequent when the large-size gangue is crushed under pressure.

Based on the deformation law, stress change, and AE energy characteristics of gangue-cement samples during loading, it can be seen that the whole process of ganguecement samples can be mainly divided into three stages. In phase I, the energy change is not obvious, mainly because the pores are compacted. The gangue does not produce any or produce a very small amount of damage. Hence, the duration of energy change is short and small. In the second stage, the energy changes violently and lasts for a long time. 


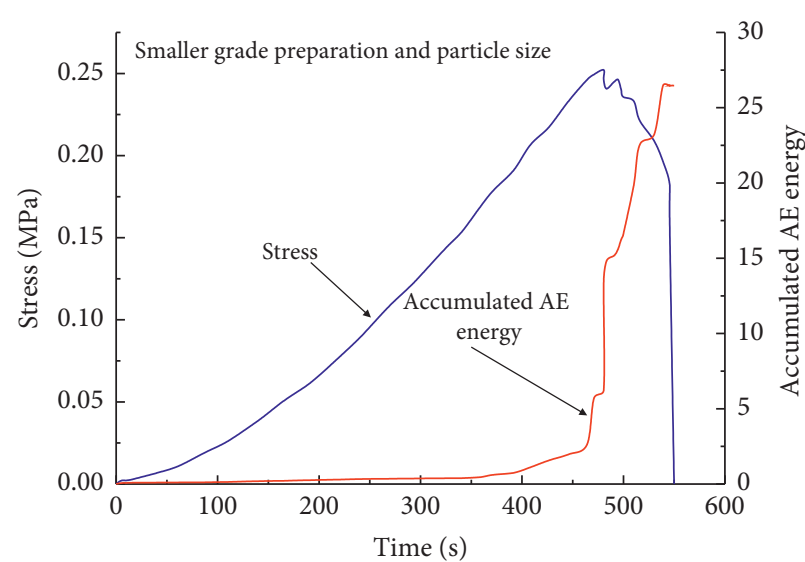

(a)

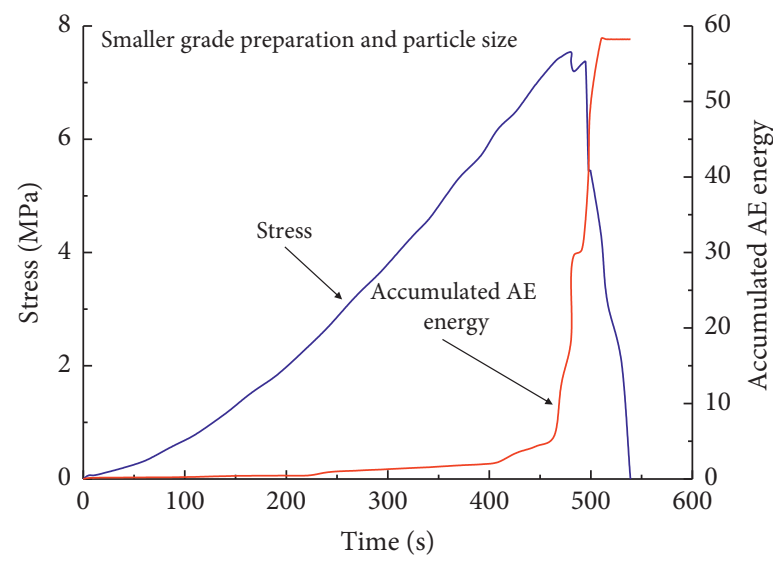

(c)

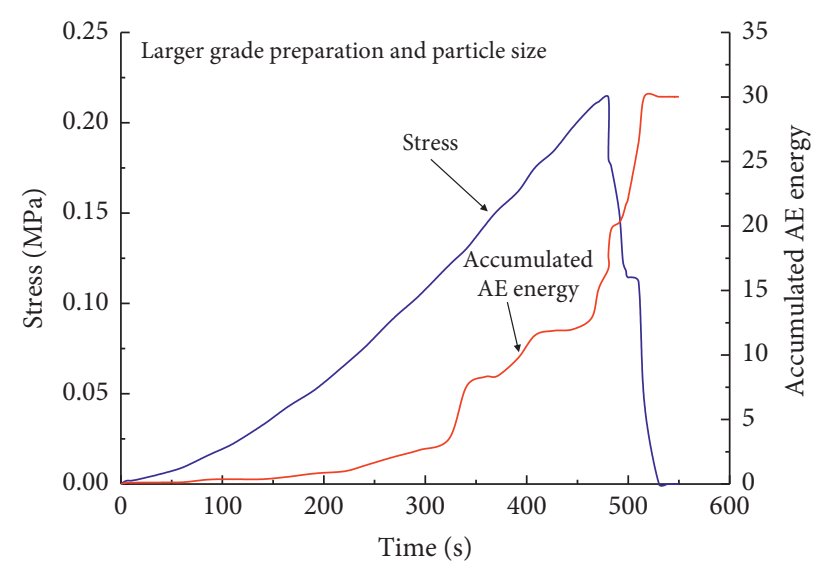

(b)

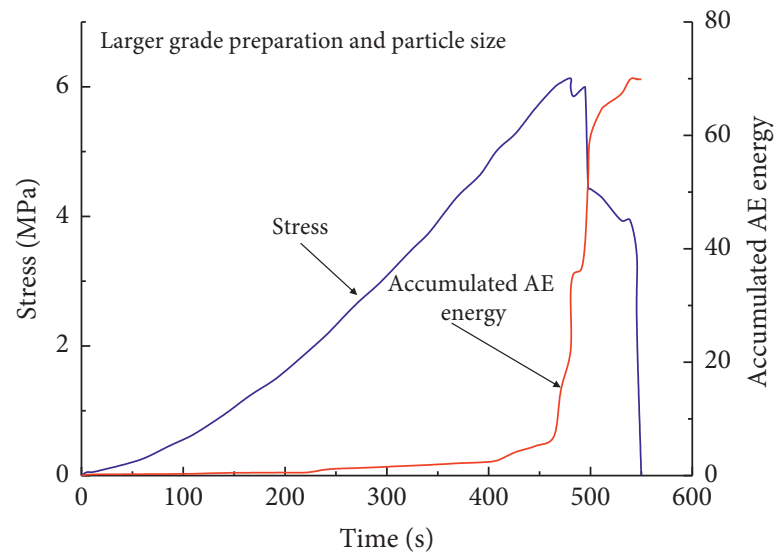

(d)

Figure 9: Time-varying characteristics of AE and stress. (a) Gangue-cement samples cured for 1 day and (b) gangue-cement samples cured for 14 days.

It is mainly because the gangue is largely broken at this stage. The gangues are squeezed with each other, and the degree of friction is severe. In the third stage, the energy change is relatively stable. It is mainly because the sample reaches a stable state at this stage, and the particle breakage is not obvious.

3.3. Fracture Propagation. A uniaxial AE test is carried out after the initial forming of the gangue-cement sample (GS), and the test results are shown in Figure 10. Under the condition of low-stress loading, fractures have appeared in the rock. The fracture propagation path is affected by the distribution of gangue, showing five distribution modes. The first type is the single surrounding gangue (I). This type is mainly because the weak performance area of the ganguecement sample makes the fracture expand before being disturbed by the gangue. Its initial expansion path is not affected by the gangue. The second is the turning surrounding the gangue (II). After stress loading, the damage and failure occur, firstly, in the area with weak performance, and then, they continue to expand. After being affected by high-performance gangue in the propagation path, the fracture occurs along a gangue-cement interface because of the significant difference of mechanical properties, resulting in path deflection. The third type is the breakthrough surrounding the gangue (III). This phenomenon is mainly manifested in that the fracture passes through the gangue. However, it is rare in fracture propagation, which is mainly caused by the differences in gangue properties. At the same time, gangue particles disintegrate during maintenance. At the same time, the close-fitting between the particles will also cause "false" apparent characteristics. The fourth type is the bifurcated surrounding gangue (IV). It is mainly manifested in the bifurcation and expansion of fractures after encountering a gangue. It is caused by the low and almost consistent strength of the interface on both sides of the gangue. The fifth type is the collapse surrounding the gangue (V). It is shown that the fractures deflect and conduct along the interface in front of the gangue and converge again behind the gangue, and cause gangue stripping and collapse. After complete curing, it mainly presents type I, and other types are rare. It is mainly because the strength of some interfaces and the cement strength reach the maximum after complete solidification. Its mechanical properties are less different from those of a gangue, resulting in less influence on stress conduction. Hence, there are fewer deflection phenomena. 

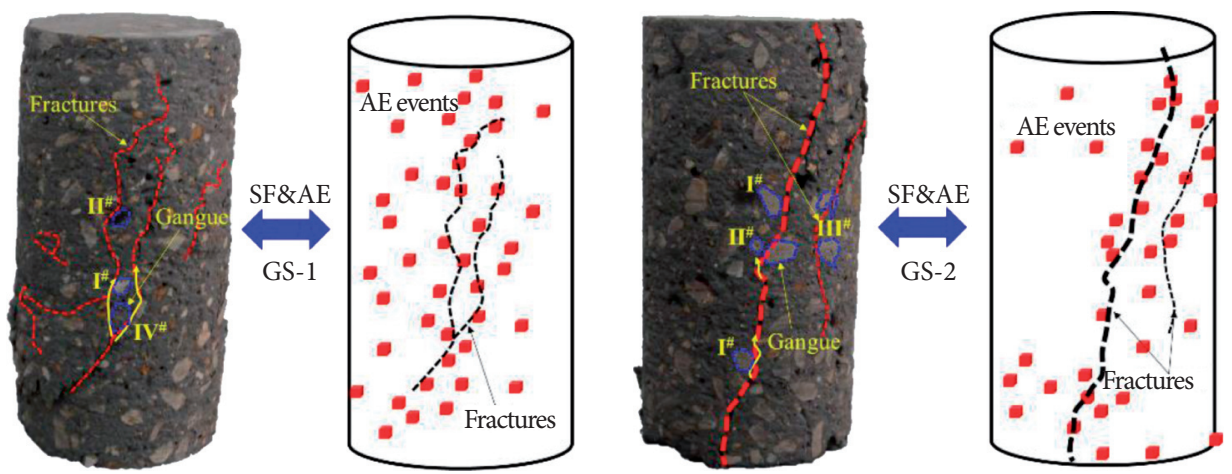

(a)
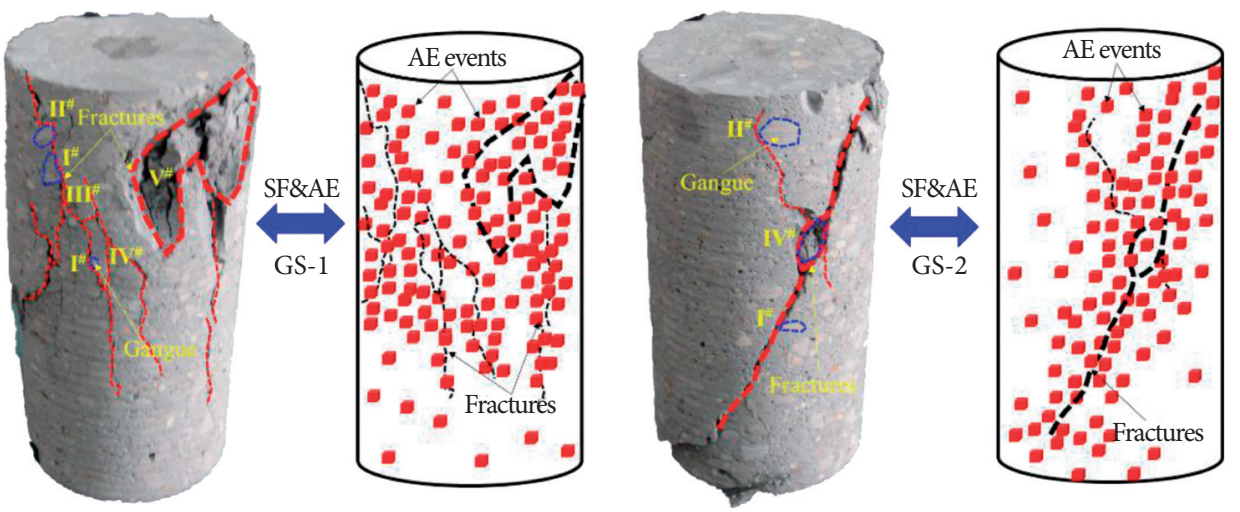

(b)
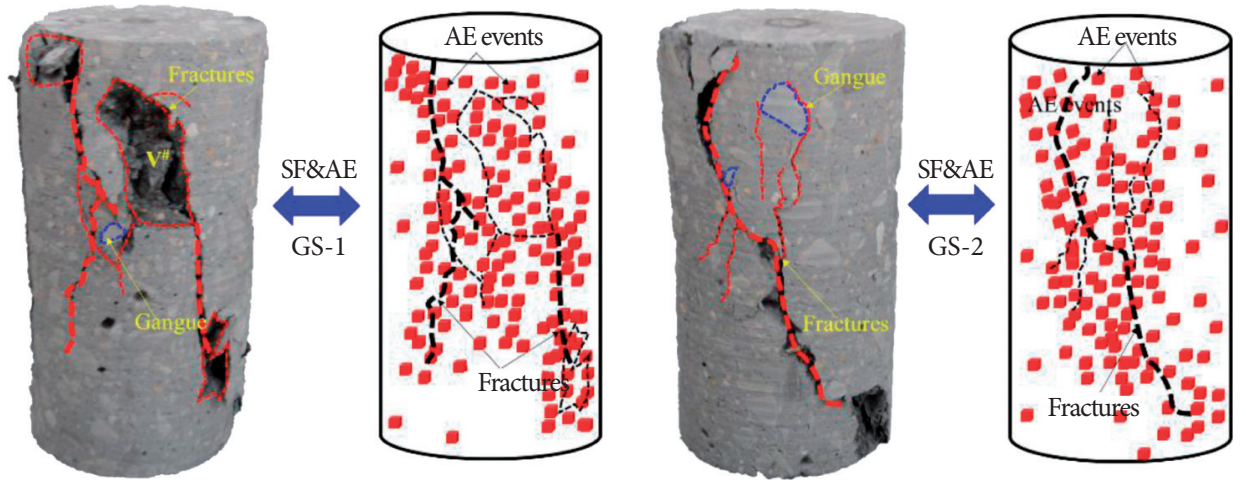

(c)

FIgURE 10: Sample fractures (SF) propagation with AE distribution. (a) Gangue-cement samples cured for 1 day, (b) gangue-cement samples cured for 10 days, and (c) gangue-cement samples cured for 14 days.

Under the condition of low-level matching, the fracture is more complex, and its $\mathrm{AE}$ events are sporadically distributed in the gangue-cement sample. In the dominant fractures, AE events meet the conditions of fracture formation. The fracture propagation process can be obtained based on the temporal and spatial distribution characteristics of AE events. Under the condition of large gradation, the fracture is relatively simple. The dominant fracture runs through the whole gangue-cement sample, and the $\mathrm{AE}$ is mainly concentrated near the dominant fracture. The reason is that the surface area of large gangue is large in the process of forming a cementitious body between a cementitious material and gangue aggregate. The structure of cement and other materials cannot be well-cemented with the gangue surface, resulting in more pores. The interface strength of the gangue cementitious material is generally lower than that of the surrounding rock. At the same time, the overall strength is generally low at the initial stage of consolidation and maintenance. It intensifies the strength difference between the gangue and cementitious material. Therefore, in the samples with large gangue, the fractures extend along the low strength weak surface under the disturbance of principal stress. Then, a single dominant fracture is formed. The interface strength formed by the small gangue and cementitious material is less different from that of the surrounding rock. Therefore, the "dominant" weak side is not obvious. Therefore, there are sporadic microfractures. The formation of main the seam is mainly type I $\backslash$ II. The reason is the 
homogeneous distribution and interference of gangue blocks. For type III, the condition for its existence is the formation of narrow cementitious materials or pores between the gangues. In the process of fracture formation, the phenomenon of pseudo breakthrough around the gangue is formed. For type IV, this form is caused by the small difference in cementitious properties on both sides of the gangue. Cause damage and rupture of the matrix under the same conditions. It can also be seen from the figure that the $\mathrm{AE}$ signal of this fracture is evenly distributed.

With the increase of the curing period, the strength of the gangue-cement sample increases, and the disintegration failure occurs under the condition of small gradation. The number of fractures is greater than that under large grading conditions. Moreover, there are many fractures around the gangue, including initial type I $\backslash$ II $\backslash$ III $\backslash$ IV. A new expansion mode $\mathrm{V}$ is produced. Under the condition of large gradation, the sample does not change greatly, and the single dominant failure fracture is the main type. Further combined with $\mathrm{AE}$ events, it can also be seen that the number of $\mathrm{AE}$ events has increased significantly compared with the previous period. The fracture is highly concentrated. Comparing the two grading conditions, the concentration of gangue-cement samples formed by large grading is greater than that formed by small grading. In other words, it forms aggregation destruction in a certain area, i.e., the large graded gangue particles form a weak surface area dominated by the interface. As the particle difference of small graded samples is low, the gangue and cementitious material are homogeneous distribution. Hence, a large number of microfractures are formed.

3.4. Spatiotemporal Distribution of Rock Failure Types. The AE system stores the obtained rock mass fracture vibration signal in the form of a waveform. Through the triggering events of more than four channels, the positioning of the rock mass fracture and the inversion of fracture type by the moment tensor are realized. The majority of scholars have done a lot of research work $[49,50]$, which will not be repeated here. According to the moment tensor inversion theory, the method proposed by Ohtsu is usually used to decompose the moment tensor in laboratory experiments [51]. The detailed method has been widely used and is only briefly described here, as shown in

$$
\left\{\begin{array}{l}
X=\frac{M_{2}-M_{3}}{M_{1}}, \\
Y=\frac{M_{1}+M_{2}+M_{3}}{3 M_{1}}, \\
Z=\frac{2 M_{1}-4 M_{2}+2 M_{3}}{3 M_{1}} .
\end{array}\right.
$$

where $M_{1}>M_{2}>M_{3}$ are the three eigenvalues of the tensor, representing the maximum couple that is independent of the selection of coordinate system. When $X>60 \%$, the source is in the shear dominant state, which is determined as a shear failure. When $60 \% X \geq 40 \%$, it is a mixed failure between shear and tension. When $X<40 \%$, it is tensile failure.

Therefore, the AE distribution characteristics under rock failure in different curing cycles are calculated as shown in Figure 11, where the red color represents shear failure, the blue color represents tensile failure, and the green color represents mixed failure. It can be found that the mechanical properties of the rock mass increase gradually with the increase of curing time. The microfracture signal of the rock mass increases with the curing time. At the same time, the difference induced by the gangue will also increase. The failure characteristics of the gangue-cement samples with different particle sizes are also significantly different. The gangue-cement sample with a small particle size always shows the characteristics of scattered microfractures. The large particle size is characterized by regional aggregation failure. All samples are mainly shear failure fractures in the whole process. In the area of gangue concentration, especially when fracture deflection occurs, there are certain tensile and mixed failures. At the same time, the tensile failure accounts for a large proportion of gangue-cement samples with large particle sizes. In other words, the gangue will change the local stress state.

To further analyze the distribution characteristics of different failure types in the whole loading process, the proportion of failure types in different periods and grading conditions is statistically obtained as shown in Figure 12. It can be found that the shear failure generally accounts for $50 \%$. At the same time, in the small particle size state, the tensile and mixed failures dominate in the early stage of curing. It is mainly affected by water, which is different from the gangue-cement sample in the dry state. With the increase of the curing period, the shear failure occupies a dominant position and shows a gradually increasing trend. After 14 days of curing, this increasing trend tends to be stable. For samples with large particle sizes, the shear fracture remains relatively stable during maintenance. It has a certain increasing trend in the initial stage. Compared with small particle size, its proportion decreases, however, it always occupies a dominant position. It can be seen that the particle size distribution characteristics of the gangue will change the failure type of the gangue-cement sample.

3.5. Failure Mechanism of Large Graded Gangue. In the gangue-cement filling body, for the gangue with large particle size, as the main aggregate, it will form an interface cementation state with cementitious particles, such as cement, as shown in Figure 13. As the gangue body is large and the surface is smooth, the particles and their surface in the concentrated area generally form regular distribution characteristics. However, because of material differences, the weak adhesion between the particles and the surface gradually accumulates with the increase of the surface area and forms a weak interface. This interface is dominated by low performance in gangue-cement samples. Under the condition of the same 

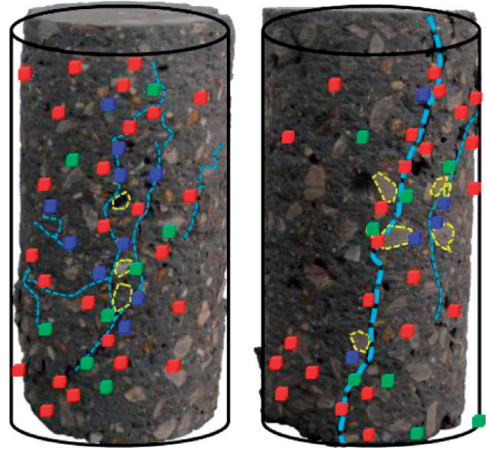

(a)

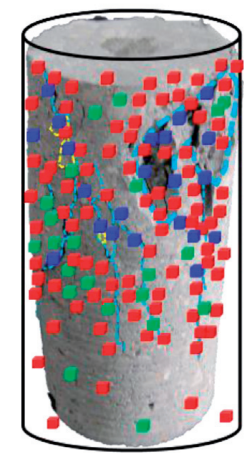

(b)

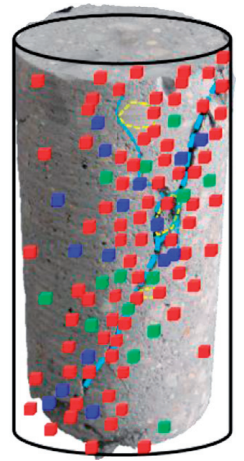

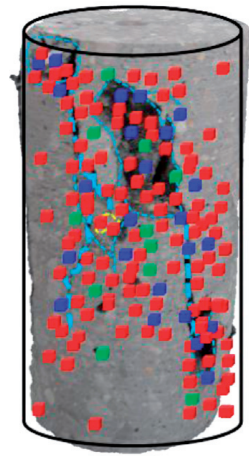

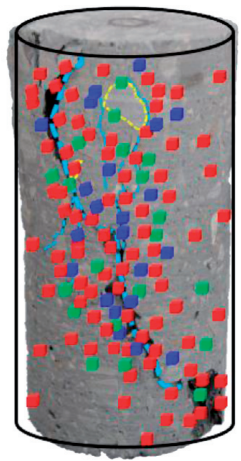

(c)

FIgUre 11: AE distribution in failure process. (a) AE distribution for 1 day, (b) AE distribution for 10 days, and (c) AE distribution for 14 days.
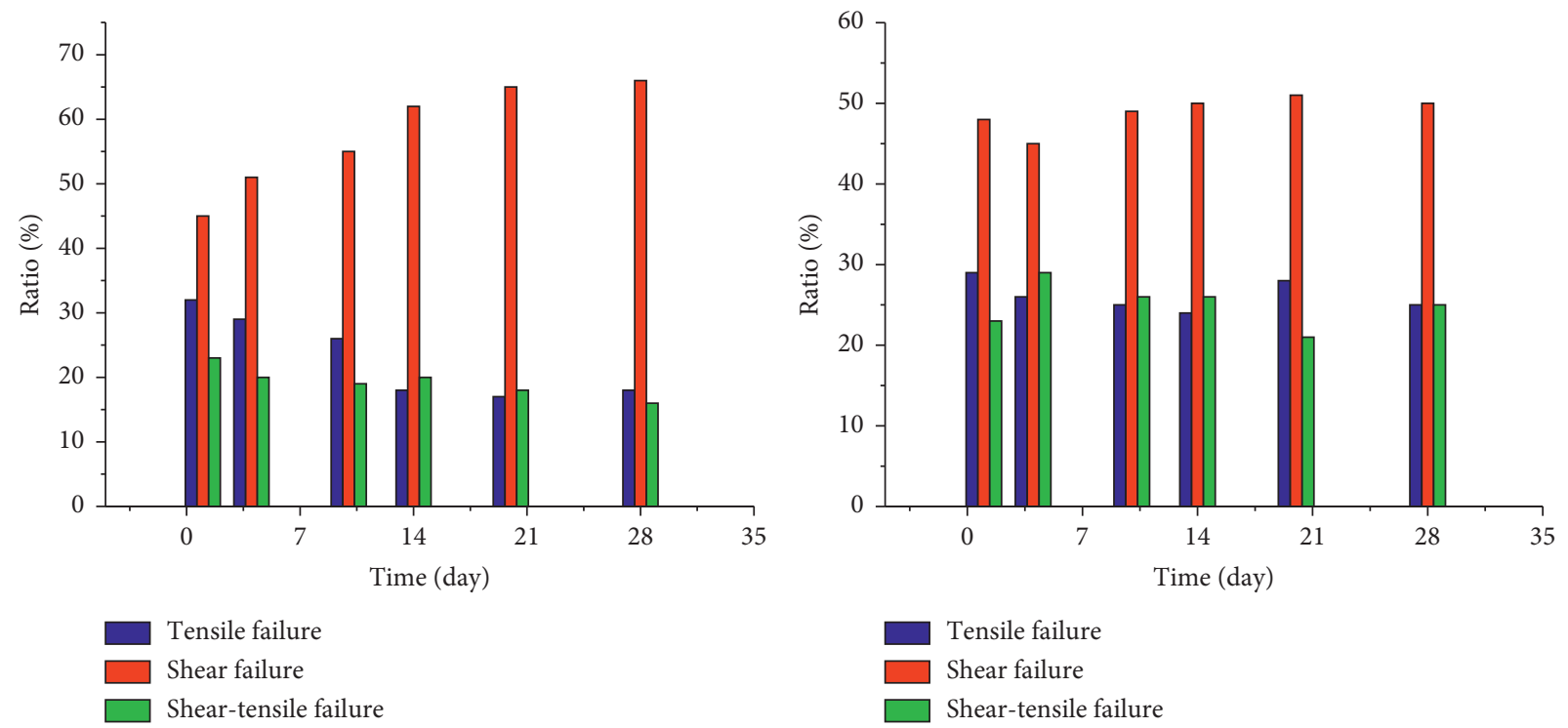

FIGURE 12: Failure type in the gangue-cement sample.

force, the damage occurs preferentially. Thus, the bond chain between the particles and the surface is damaged, resulting in different degrees of damage aggregation and fracture propagation.

Because of the properties of gangue block and the distribution characteristics of cementitious particles, the gangue hinders the expansion of stress fractures in the horizontal plane in front of the gangue. However, it cannot affect its extension in the upper and lower rocks of the gangue. At this time, the fracture will expand around the gangue in the longitudinal direction as shown in Figure 13. Firstly, when the interfacial adhesion between the cementitious particles and the gangue is strong, as there is a weaker area near the gangue, the fracture will continue to expand along the original channel and forms a fracture of type I. Secondly, when the weak surface formed at the interface between the cementitious particles and the gangue exists in a single direction, the stress-induced fracture propagates along the weak surface and produces a fracture of type II. Thirdly, when the gangue has a weak surface (or initial damage fracture), the strength of the weak surface is generally lower than that at other positions. Then, the stress causes material damage in a certain direction, induces the fracture to penetrate the gangue, and expands to produce a fracture of type III. Moreover, when the low adhesion between the gangue and the cementitious particles has multidirectionality, the weak surface exists on both surfaces. The stress causes material damage in both directions. Then, the fracture is induced to expand in two directions and extend forward at a certain angle, resulting in a type IV fracture. Finally, on the premise that the gangue has weak surfaces in two directions when there is weak material behind the gangue or causes typical stress concentration, the fracture will continue to expand along the gangue surface. Form a package expansion mode, merge, and extend inward, resulting in a type $\mathrm{V}$ fracture. 


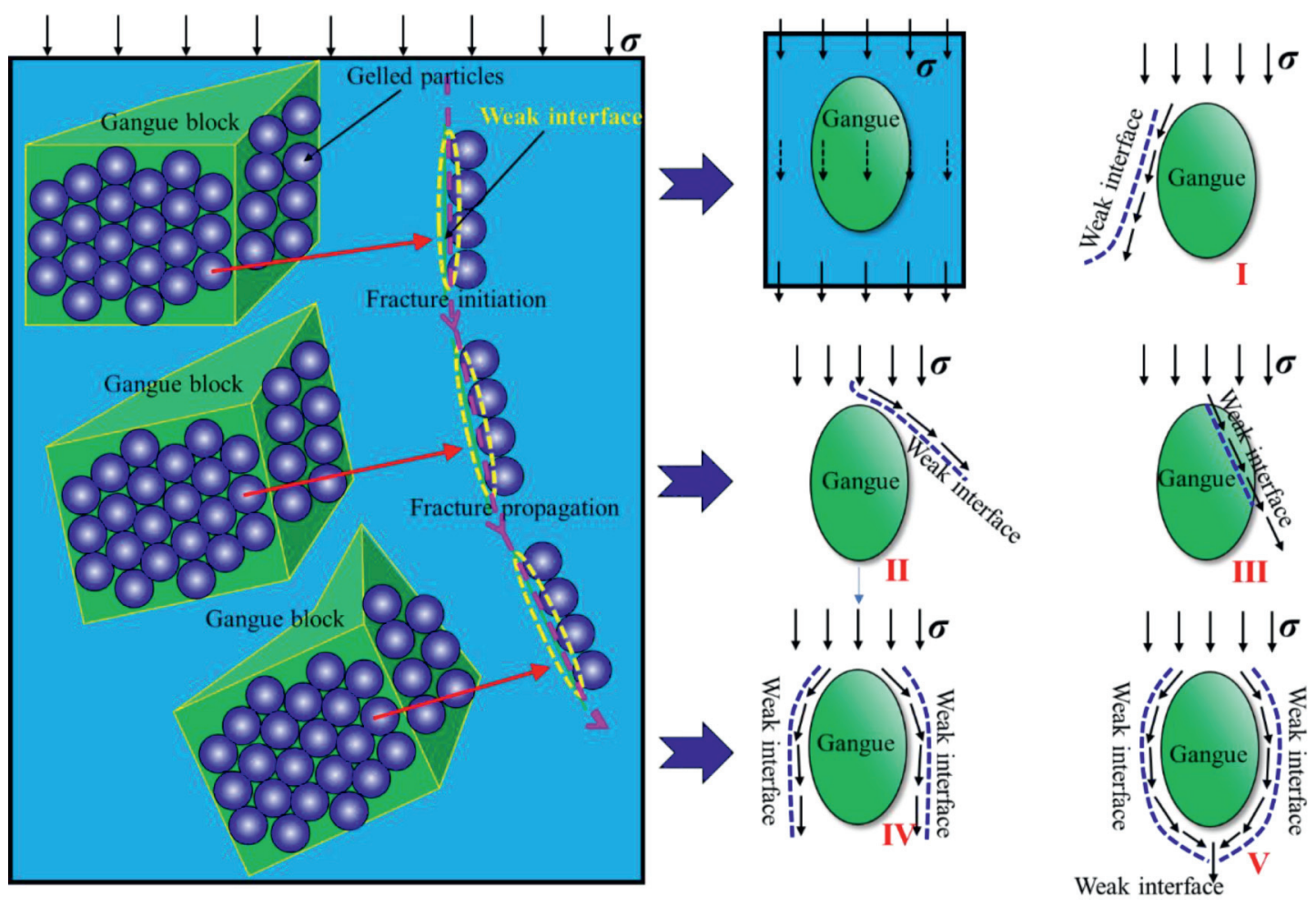

Figure 13: Dynamic load deflection induced by gangue particles.

The application of gangue filling materials should always be based on a reasonable ratio. Especially, the control of gangue particle size and gradation. At the same time, attention should be paid to the uniform distribution of gangue and other cementitious materials to avoid V-shaped characteristics. This phenomenon will directly affect the strength performance of the filling body. The gangue with medium and coarse particle size acts as an aggregate in the filling material, and they form a support network system with each other. Under the action of external force, the focus will fall on the fulcrum of the coarse gangue group. As the gangue is a sedimentary rock, it has a relatively complete cleavage and a developed layered structure. If the stress direction is parallel to the bedding direction, the strength is small. In the filling material, the gangue is arranged irregularly. If the stress direction of the force point of a gangue particle is parallel to the bedding direction or the included angle is small, it is easy to break. The strength of the whole filling material is reduced. The uncontrollable damage of the filling body is induced in the technical implementation of the Retaining Roadway along the goaf to control the disaster and the filling goaf to prevent surface subsidence. The application of gangue should be fully mixed under homogeneous and uniform particles. The structure and roughness of the aggregate surface also play an important role in the compressive performance of the cement paste. The surface of the gangue is relatively smooth. It is not easy to form a stable cementation structure in the interface transition zone between the slurry and aggregate, which is not conducive to enhancing the compressive strength of the materials.

\section{Conclusion}

In this study, the experimental research on the mechanical properties and rock failure characteristics of the ganguecement filling materials under different factors is carried out by making the gangue filling materials with different properties. It is of great significance for underground engineering filling to control surrounding rock subsidence and failure. The main conclusions are as follows:

(1) The filling body formed by the gangue material is affected by the amount of cementitious material and R-WCM. Among them, the greater the amount of cementitious material, the greater the early strength value. The increase of the cementitious material will increase the cost of filling and mining. The amount of cementitious material should be determined according to the strength requirements in combination with the actual situation. With the increase of the water binder ratio, the early strength decreases. However, the water binder ratio cannot be reduced blindly because its reduction will reduce the slump and make it difficult to transport the slurry.

(2) The compression deformation of the gangue backfill can be roughly divided into three stages. The first one is the stage of linear deformation. The compressive deformation of the gangue increases linearly with the 
increase of vertical stress. The second one is the stage of block compression. The crushed gangue further fills and compacts the small voids. The third one is characterized by gentle stability. The compressive deformation of the gangue tends to be gentle in a linear manner with the increase of stress. The three stages are affected by single particle size gangue and its grading characteristics. With the increase of the fine gangue rate, the fine aggregate filled into the pores increases, and its strength is improved.

(3) At the initial stage of gangue cementation, the rock mass is damaged and damaged by the load. The gangue is arranged irregularly. If the stress direction of the force point of a gangue particle is parallel to the bedding direction or the included angle is small, damage accumulation is easy to occur. Fracture propagation induced in different paths under the influence of gangue distribution has five distribution modes: single, turning, breakthrough, bifurcated, and collapsed surrounding gangue. Under the condition of small gradation, the disintegration failure of the ganguecement sample occurs, and the number of fractures is significantly greater than that under the condition of large gradation. Under the condition of large gradation, the sample does not change greatly, and it is dominated by a single dominant failure fracture.

(4) The whole process of the gangue-cement sample is dominated by shear failure fractures. In the area of gangue concentration, especially when fracture deflection occurs, there are certain tensile and mixed failures. At the same time, the particle size distribution characteristics will change the stressstate concentration characteristics that can change the failure type of rock. The structure and roughness of the aggregate surface also play an important role in the compressive performance of the cement paste. The phenomenon of the $\mathrm{V}$-shaped waste winding should be avoided when applying materials.

\section{Data Availability}

The data used to support the findings of this study are included within the article.

\section{Conflicts of Interest}

The authors declare that they have no conflicts of interest.

\section{Acknowledgments}

This work was conducted with support from the Natural Science Foundation of Anhui Province (grant nos. 2008085ME145 and 2108085QE209) and the Key Research and Development Plan of Huainan City (grant no. 2021A05).

\section{References}

[1] H. Luan, Y. Jiang, H. Lin, and Y. Wang, "A new thin seam backfill mining technology and its application," Energies, vol. 10, no. 12, 2017.

[2] W. Y. Lv, K. Guo, J. H. Yu, X. F. Du, K. Feng, and B. Tan, "Surrounding rock movement of steeply dipping coal seam using backfill mining," Shock and Vibration, vol. 2021, Article ID 5574563, 17 pages, 2021.

[3] Z. X. Liu, S. N. Dong, H. Wang, C. H. Zhao, and Z. F. Zhou, "Influences on the performance of cement-based grout used to reform the upper Middle Ordovician limestone in Hanxing mining area," Arabian Journal of Geosciences, vol. 14, pp. 1-15, 2021.

[4] W. Mu, D. Wang, L. Li et al., "Cement flow in interaction rock fractures and its corresponding new construction process in slope engineering," Construction and Building Materials, vol. 303, Article ID 124533, 2021.

[5] W. Mu, L. Li, T. Yang, G. Yu, and Y. Han, "Numerical investigation on a grouting mechanism with slurry-rock coupling and shear displacement in a single rough fracture," Bulletin of Engineering Geology and the Environment, vol. 78, no. 8, pp. 6159-6177, 2019.

[6] D. L. Yang, J. M. Li, Y. L. Huang, T. Q. Song, H. D. Gao, and M. Qiao, "Research on migration law of Mn in mudstone floor in the goaf under coupling conditions of seepage and stress," Polish Journal of Environmental Studies, vol. 29, pp. 939-950, 2020.

[7] J. Z. Li, M. Zhang, Y. Li, and H. Hu, "Surrounding rock control mechanism in the gob-side retaining entry in thin coal seams, and its application," Journal of the South African Institute of Mining and Metallurgy, vol. 118, pp. 471-480, 2018.

[8] W. Mu, L. Li, Z. Guo, Z. Du, and S. Wang, "Novel segmented roadside plugging-filling mining method and overlying rock mechanical mechanism analyses," Energies, vol. 12, no. 11, 2019.

[9] J. G. Liu, X. W. Li, and T. He, "Application status and prospect of backfill mining in Chinese coal mines," Journal of China Coal Society, vol. 45, pp. 141-150, 2020.

[10] T. B. Zhao, Z. Y. Fu, and G. Li, "In situ investigation into fracture and subsidence of overburden strata for solid backfill mining," Arabian Journal of Geosciences, vol. 11, pp. 1-11, 2018.

[11] J. Sun, X. Liu, and T. Ren, "Overburden stability of an inclined backfill stope in the context of the nonlinear elastic mechanical properties of the backfill body," Environmental Earth Sciences, vol. 78, pp. 725-733, 2019.

[12] Q. D. Li, G. R. Feng, Y. X. Guo et al., "The dosage of superplasticizer in cemented coal waste backfill material based on response surface methodology," Advances in Materials Science and Engineering, vol. 2019, Article ID 5328523, 8 pages, 2019.

[13] W. Yin, J. Q. Wang, X. M. Bai, W. J. Sun, and Z. Y. Zhou, "Strata behavior and control strategy of backfilling collaborate with caving fully- mechanized mining," Open Geosciences, vol. 12, pp. 703-717, 2020.

[14] J. Sun, B. Li, G. M. Zhao, and L. G. Wang, "Sensitivity analysis of overburden water-resistant strata stability based on the mechanical properties of backfill body," Arabian Journal of Geosciences, vol. 13, pp. 1105-1110, 2020.

[15] W. Yin, X. M. Bai, J. K. Wu, R. Zhang, C. Liu, and Y. X. Tang, "Mechanical analysis of basic roof fracture mechanism and feature in coal mining with partial gangue backfilling," Open Geosciences, vol. 12, pp. 904-918, 2020. 
[16] Y. X. Guo, Y. H. Zhao, G. R. Feng, H. Y. Ran, and Y. J. Zhang, "Study on damage size effect of cemented gangue backfill body under uniaxial compression," Chinese Journal of Rock Mechanics and Engineering, vol. 40, 2021.

[17] Q. Zhang, J. Zhang, Z. Wu, Y. Chen, K. Xia, and H. Liu, "Function relational expression of backfilled body's density and its influencing factors," Energy Sources, Part A: Recovery, Utilization, and Environmental Effects, vol. 43, no. 18, pp. 2218-2234, 2021.

[18] C. Ma, L. Y. Zhang, B. Li, X. B. Mao, and J. F. Zhang, "Constitutive model of solid backfill materials and numerical simulation of overburden movement and deformation in backfill mining," Shock and Vibration, vol. 2021, Article ID 6619532, 14 pages, 2021.

[19] W. Li, Y. L. Huang, H. D. Gao, J. M. Li, Z. Y. Ruan, and T. Q. Song, "Study on acoustic emission characteristics of guague of different graduations during confined compression," Journal of Mining \& Safety Engineering, vol. 37, pp. 154-161+168, 2020.

[20] Q. Sun, N. Zhou, W. J. Song, X. Zhao, and D. Z. Kong, "Risk assessment and prevention of surface subsidence under buildings by cemented paste filling and strip mining methods: a case study," Advances in Civil Engineering, vol. 2021, Article ID 9965279, 10 pages, 2021.

[21] M. S. Awadh and K. Nejbert, "Polymetallic sulfide ores hosted in Late Permian carbonate at the Alanish locality, northern Iraq: petrography and mineral chemistry," Arabian Journal of Geosciences, vol. 9, 2016.

[22] W. Liu, J. Chen, Z. Guo, H. Yang, W. Xie, and Y. D. Zhang, "Mechanical properties and damage evolution of cemented coal gangue-fly ash backfill under uniaxial compression: effects of different curing temperatures," Construction and Building Materials, vol. 305, Article ID 124820, 2021.

[23] W. Liu, Z. Guo, C. Wang, and S. Niu, "Physico-mechanical and microstructure properties of cemented coal Gangue-Fly ash backfill: effects of curing temperature," Construction and Building Materials, vol. 299, Article ID 124011, 2021.

[24] Z. Huang, L. Zhang, Z. G. Ma, and H. L. Kong, "Study on the mechanical relationship among the backfilling mining support, roof rock beam, and gangue filling body in comprehensive mechanized filling mining process," Advances in Civil Engineering, vol. 2020, Article ID 8824735, 15 pages, 2020.

[25] J. H. Potgieter and S. S. Potgieter, "Mining backfill formulations from various cementitious and waste materials," Indian Concrete Journal, vol. 77, pp. 1071-1075, 2003.

[26] Y. L. Huang, J. X. Zhang, and J. Du, "Time-dependence of backfilling body in fully mechanized backfilling mining face," Journal of China University of Mining and Technology, vol. 41, pp. 697-701, 2012.

[27] J. M. Xu, J. X. Zhang, Y. L. Huang, and F. Ju, "Experimental research on the compress deformation characteristic of wastefly ash and its application in backfilling fully mechanized coal mining technology," Journal of Mining \& Safety Engineering, vol. 28, pp. 158-162, 2011.

[28] X. Liu, S. H. Tu, D. Y. Hao et al., "Deformation law and control measures of gob-side entry filled with gangue in deep gobs: a case study," Advances in Materials Science and Engineering, vol. 2021, Article ID 9967870, 13 pages, 2021.

[29] W. Y. Qi, J. X. Zhang, N. Zhou, Z. Y. Wu, J. Zhang, and H. Haddadpour, "Mechanism by which backfill body reduces amount of energy released in deep coal mining," Shock and Vibration, vol. 2019, Article ID 8253269, 14 pages, 2019.

[30] M. Li, A. Li, J. Zhang, Y. Huang, and J. Li, "Effects of particle sizes on compressive deformation and particle breakage of gangue used for coal mine goaf backfill," Powder Technology, vol. 360, pp. 493-502, 2020.

[31] J. M. Li, Y. L. Huang, W. Zhai et al., "Experimental Study on Acoustic Emission of Confined Compression of Crushed Gangue under Different Loading Rates: Disposal of Gangue Solid Waste," Sustainability, vol. 12, no. 1, 2020.

[32] H. Wang, J. Jiao, Y. M. Wang, and W. S. Du, "Feasibility of using gangue and fly ash as filling slurry materials," Processes, vol. 6, 2018.

[33] Q. Zhang, Z. J. Wang, J. X. Zhang et al., "Integrated green Mining Technology of 'coal Mining-Gangue WashingBackfilling-Strata Control-System Monitoring'-Taking Tangshan Mine as a Case Study," Environmental science and pollution research international, 2021.

[34] P. Huang, A. J. S. Spearing, J. Feng, K. V. Jessu, and S. Guo, "Effects of solid backfilling on overburden strata movement in shallow depth longwall coal mines in West China," Journal of Geophysics and Engineering, vol. 15, no. 5, pp. 2194-2208, 2018.

[35] S. Chen, Z. Du, Z. Zhang, D. Yin, F. Feng, and J. Ma, "Effects of red mud additions on gangue-cemented paste backfill properties," Powder Technology, vol. 367, pp. 833-840, 2020.

[36] S. J. Chen, Z. W. Du, Z. Zhang, H. W. Zhang, Z. G. Xia, and F. Feng, "Effects of chloride on the early mechanical properties and microstructure of gangue-cemented paste backfill," Construction and Building Materials, vol. 235, 2020.

[37] L. G. Tam, H. Liu, C. A. Tang, P. K. K. Lee, and Y. Tsui, “On tension failure of 2-D rock specimens and associated acoustic emission," Rock Mechanics and Rock Engineering, vol. 38, no. 1, pp. 1-19, 2005.

[38] P. Ganne, A. Vervoort, and M. Wevess, "Quantifcation of prebreak brittle damage correlation between acoustic emission andobserved micro-fracture," International Journal of Mechanics \&Mining Sciences, vol. 44, no. 5, pp. 720-729, 2007.

[39] T. Qin, H. Sun, H. Liu et al., "Experimental study on mechanical and acoustic emission characteristics of rock samples under different stress paths," Shock and Vibration, vol. 2018, Article ID 4813724, 9 pages, 2018.

[40] F. Yang, D. Hu, H. Zhou, M. Teng, M. Lan, and Q. Teng, "Post-fatigue properties of high-strength concrete subjected to coupled 3D fatigue-static loading," Construction and Building Materials, vol. 306, Article ID 124879, 2021.

[41] J. Zhu and Y. Song, "Research on fatigue damage of concrete under biaxial compressive loading using ultrasonic velocity method," Journal of Rock Mechanics and Engineering, vol. 23, no. 13, pp. 2230-2234, 2004.

[42] M. Chen, S. Q. Yang, R. G. Pathegama et al., "Fracture processes of rock-like specimens containing nonpersistent fissures under uniaxial compression," Energies, vol. 12, no. 1, 2019.

[43] J. M. Li, Y. L. Huang, W. Y. Qi, G. Q. Kong, and T. Q. Song, "Loose gangues backfill body's acoustic emissions rules during compaction test: based on solid backfill mining," Computer Modeling in Engineering and Sciences: Computer Modeling in Engineering and Sciences, vol. 115, no. 1, pp. 85-103, 2018.

[44] T. Y. Qi and G. R. Feng, "Resistivity and A. E response characteristics in the failure process of CGB under uniaxial loading," Advances in Materials Science and Engineering, vol. 2017, Article ID 7857590, 11 pages, 2017.

[45] N. Zhou, H. B. Ma, S. Y. Ouyang, D. Germain, and T. Hou, "Influential factors in transportation and mechanical properties of aeolian sand-based cemented filling material," Minerals, vol. 9, no. 2, 2019. 
[46] A. Das, C. Jayashree, and B. V. S. Viswanadham, "Effect of randomly distributed geofibers on the piping behaviour of embankments constructed with fly ash as a fill material," Geotextiles and Geomembranes, vol. 27, no. 5, pp. 341-349, 2009.

[47] A. Q. Zhang, "Application of Taibo theory in mineral aggregate gradation design," Journal of Shandong Institute of building materials, vol. 14, no. 2, pp. 140-142, 2000.

[48] Y. Zhao, C. L. Wang, and J. Bi, "Analysis of fractured rock permeability evolution under unloading conditions by the model of elastoplastic contact between rough surfaces," Rock Mechanics and Rock Engineering, vol. 53, no. 12, pp. 57955808, 2020.

[49] G. Kwiatek, E. M. Charalampidou, G. Dresen, and S. Stanchits, "An improved method for seismic moment tensor inversion of acoustic emissions through assessment of sensor coupling and sensitivity to incidence angle," International Journal of Rock Mechanics and Mining Sciences, vol. 65, no. 1, pp. 153-161, 2014.

[50] I. V. Rodriguez, S. Stanchits, and J. Burghardt, "Data-Driven, in situ, relative sensor calibration based on waveform fitting moment tensor inversion," Rock Mechanics and Rock Engineering, vol. 50, no. 4, pp. 891-911, 2017.

[51] M. Ohtsu, "Acoustic emission theory for moment tensor analysis," Research in Nondestructive Evaluation, vol. 6, no. 3, pp. 169-184, 1995. 